MATHEMATICS OF COMPUTATION

Volume 74, Number 251, Pages 1097-1116

S 0025-5718(04)01700-4

Article electronically published on July 16, 2004

\title{
POINTWISE ERROR ESTIMATES \\ OF THE LOCAL DISCONTINUOUS GALERKIN METHOD FOR A SECOND ORDER ELLIPTIC PROBLEM
}

\author{
HONGSEN CHEN
}

\begin{abstract}
In this paper we derive some pointwise error estimates for the local discontinuous Galerkin (LDG) method for solving second-order elliptic problems in $R^{N}(N \geq 2)$. Our results show that the pointwise errors of both the vector and scalar approximations of the LDG method are of the same order as those obtained in the $L^{2}$ norm except for a logarithmic factor when the piecewise linear functions are used in the finite element spaces. Moreover, due to the weighted norms in the bounds, these pointwise error estimates indicate that when at least piecewise quadratic polynomials are used in the finite element spaces, the errors at any point $z$ depend very weakly on the true solution and its derivatives in the regions far away from $z$. These localized error estimates are similar to those obtained for the standard conforming finite element method.
\end{abstract}

\section{INTRODUCTION}

The aim of this paper is to derive some pointwise error estimates of the local discontinuous Galerkin (LDG) method for solving the second order elliptic problems. The LDG method is a discontinuous Galerkin method in the mixed formulation and was introduced by Cockburn and Shu [15]. The LDG method has been used for solving different types of differential equations including elliptic equations ([14]). A rigorous error analysis in the $L^{2}$ norm for the LDG method can be found in Castillo, Cockburn, Perugia and Shötzau in [9]. To describe our results, we state that the best error estimate in the $L^{2}$ norm obtained in $[9$ for the LDG method is the following:

$$
\left\|p-p_{h}\right\|_{L^{2}(\Omega)}+h\left\|\mathbf{u}-\mathbf{u}_{h}\right\|_{L^{2}(\Omega)} \leq C h^{1+r}\|p\|_{H^{1+r}(\Omega)} .
$$

Here, $\Omega \subset R^{N}(N \geq 2),(p, \mathbf{u}) \in H^{1+r}(\Omega) \times H^{r}(\Omega)^{N}$ and $\left(p_{h}, \mathbf{u}_{h}\right)$ are the true and approximate solutions of the LDG method, respectively, and $r \geq 1$ is the order of the polynomials used in the finite element space. The pointwise error estimates for $p-p_{h}$ and $\mathbf{u}-\mathbf{u}_{h}$ obtained in this paper take the following form (see Theorems 3.1

Received by the editor December 7, 2003 and, in revised form, February 21, 2004.

2000 Mathematics Subject Classification. Primary 65N30, 65N15, 65N12; Secondary 41A25, 35B45, 35J20.

Key words and phrases. Local discontinuous Galerkin method, pointwise error estimate, maximum norm, elliptic problem. 
and 4.1): for any $z \in \Omega$ and $0 \leq s \leq r-1$,

$$
\begin{aligned}
& \left|\left(p-p_{h}\right)(z)\right|+h\left|\left(\mathbf{u}-\mathbf{u}_{h}\right)(z)\right| \\
& \quad \leq C|\ln h|^{\bar{s}}\left(\left\|\sigma_{z, h}^{s}\left(p-Q^{h} p\right)\right\|_{L^{\infty}(\Omega)}+h\left\|\sigma_{z, h}^{s}\left(\mathbf{u}-\Pi^{h} \mathbf{u}\right)\right\|_{L^{\infty}(\Omega)}\right),
\end{aligned}
$$

where $\sigma_{z, h}^{s}(x)=h^{s} /(|x-z|+h)^{s}, \bar{s}=0$ if $0 \leq s<r-1$ and $\bar{s}=1$ if $s=r-1$, and $Q^{h}$ and $\Pi^{h}$ are, respectively, $L^{2}$ projections into the scalar and vector finite element spaces. This result, along with the approximation properties of the finite element spaces, indicates that the pointwise errors of both the vector and scalar solutions of the LDG method are of the same order as the corresponding errors measured in the $L^{2}$ norm, except the logarithmic factor $|\ln h|$ for the finite element method with the first order approximation $(r=1)$. Due to the weight function $\sigma_{z, h}$, we can see that, when at least piecewise quadratic polynomials are used in the finite element spaces, these errors at any point $z$ are dependent on the true solution mainly at points near $z$ and their dependences on the true solution in the regions far away from $z$ is weak. These localized error estimates are similar to those obtained by Schatz 22] for the standard continuous Galerkin method and by Demlow [16] for the standard conforming mixed finite element method. We also mention that some localized pointwise error estimates for a discontinuous Galerkin method in its primal formulation have been obtained in Chen and Chen [11]. We note that the estimate (1.2) reduces to the following global maximum norm error estimate when $s=0$ :

$\left\|p-p_{h}\right\|_{L^{\infty}(\Omega)}+h\left\|\mathbf{u}-\mathbf{u}_{h}\right\|_{L^{\infty}(\Omega)} \leq C|\ln h|^{\bar{r}}\left(\left\|p-Q^{h} p\right\|_{L^{\infty}(\Omega)}+h\left\|\mathbf{u}-\Pi^{h} \mathbf{u}\right\|_{L^{\infty}(\Omega)}\right)$, where $\bar{r}=0$ if $r>1$ and $\bar{r}=1$ if $r=1$. In a forthcoming paper, we will derive pointwise posterior error estimates for the LDG method so that efficient adaptive algorithms can be developed for local grid refinements.

The rest of the paper is organized in the following way. In Section 2, we define notation and the local discontinuous Galerkin method and collect some known results. In Section 3, we state and prove the pointwise error estimate for the scalar approximation. The corresponding pointwise error estimate for the vector solution is in the last section.

\section{Preliminaries}

For the sake of simplicity, we consider the following model elliptic problem with homogeneous Dirichlet boundary condition:

$$
-\Delta p=f \quad \text { in } \Omega, \quad p=0 \text { on } \partial \Omega,
$$

where $\Omega \in R^{N}(N \geq 2)$ is a bounded domain with smooth boundary $\partial \Omega$ and $f$ is a given function.

We shall use the standard notation for the Sobolev spaces and their norms. For any subdomain $D \subset \Omega$, nonnegative integer $\ell$ and real number $1 \leq t \leq \infty$, denote the Sobolev spaces by $W^{\ell, t}(D)=\left\{v:\|v\|_{W^{\ell, t}(D)}<\infty\right\}$ with

$$
\|v\|_{W^{\ell, t}(D)}=\left(\sum_{i=0}^{\ell}|v|_{W^{i, t}(D)}^{2}\right)^{1 / 2},|v|_{W^{i, t}(D)}=\left(\sum_{|\alpha|=i} \int_{D}\left|\frac{\partial^{\alpha} v(x)}{\partial x^{\alpha}}\right|^{t} d x\right)^{1 / t} .
$$

We also adopt the usual notation for $H^{\ell}(D)=W^{\ell, 2}(D)$ and $L^{t}(D)=W^{0, t}(D)$. Denote by $(\cdot, \cdot)$ the inner product in $L^{2}(\Omega)$ given by $(u, v)=\int_{\Omega} u(x) v(x) d x$. For 
$\ell \geq 0$ and $1 \leq t<\infty$, the negative norm $\|\cdot\|_{W^{-\ell, t}(D)}$ is defined as follows:

$$
\|v\|_{w^{-\ell, t}(D)}=\sup _{\varphi \in C_{0}^{\infty}(D)} \frac{(v, \varphi)}{\|\varphi\|_{W^{\ell, t^{\prime}}(D)}}
$$

where $1 / t+1 / t^{\prime}=1$ and $C_{0}^{\infty}(D)$ denotes the space of functions with continuous derivatives of arbitrary order and compact supports in $D$. We write $H^{-\ell}(D)=$ $W^{-\ell, 2}(D)$.

To introduce the discontinuous Galerkin method, let $\mathcal{J}_{h}$ denote a partition of the domain $\Omega$ into a finite collection of $N_{h}$ open subdomains $K_{j}, j=1,2, \cdots, N_{h}$, such that

$$
\bar{\Omega}=\bigcup_{K_{j} \in \mathcal{J}_{h}} \bar{K}_{j}, \quad \text { and } K_{i} \cap K_{j}=\emptyset, \quad \text { if } i \neq j .
$$

We assume that the partition $\mathcal{J}_{h}$ is globally shape regular. To be more precise, let $B_{\rho}(z)$ denote the ball centered at $z \in R^{N}$ and with radius $\rho$ and set

$$
h_{K}=\operatorname{diam}(K), \quad h=\max _{K \in \mathcal{J}_{h}} h_{K}, \quad \rho_{K}=\max \left\{\rho: B_{\rho}(z) \subset K, z \in K\right\} .
$$

Then, there are constants $C_{1}>0$ and $C_{2}>0$ such that

$$
h \leq C_{1} \min _{K \in \mathcal{J}_{h}} h_{K}, \quad \frac{h_{K}}{\rho_{K}} \leq C_{2}, \quad \forall K \in \mathcal{J}_{h} .
$$

We note that the so-called "hanging nodes" are allowed in the partition $\mathcal{J}_{h}$. Furthermore let $\Gamma_{h}$ denote the set of $(N-1)$-dimensional open subsets $e_{j}, j=1,2, \cdots, N_{h}^{e}$, such that

and let

$$
\bigcup_{j=1}^{N_{h}} \partial K_{j}=\bigcup_{j=1}^{N_{h}^{e}} \bar{e}_{j}, \quad \text { and } \quad e_{i} \cap e_{j}=\emptyset, \quad \text { if } \quad i \neq j
$$

$$
\Gamma_{h}^{0}=\left\{e \in \mathcal{J}_{h}: e \cap \partial \Omega=\emptyset\right\} .
$$

We assume that for each $e \in \Gamma_{h}^{0}$, there are $K, K^{\prime} \in \mathcal{J}_{h}$ such that $e \subset \partial K \cap \partial K^{\prime}$ and define $h_{e}=\left(h_{K}+h_{K^{\prime}}\right) / 2$. If $e \in \Gamma_{h} \backslash \Gamma_{h}^{0}$, then there is a $k \in \mathcal{J}_{h}$ such that $e \in \partial K$ and we define $h_{e}=h_{K}$. For each $K \in \mathcal{J}_{h}$, let $n_{K} \in R^{N}$ denote the unit outward normal vector on $\partial K$.

We now introduce notation for function spaces associated with the partition $\mathcal{J}_{h}$. For $\ell \geq 0$ and $1 \leq t \leq \infty$, define the discontinuous Sobolev space

$$
W_{h}^{\ell, t}(D)=\left\{v: v \in W^{\ell, t}(K) \text { for each } K \in \mathcal{J}_{h} \text { and }\|v\|_{W_{h}^{\ell, t}(D)}<\infty\right\}
$$

equipped with the broken norm

$$
\|v\|_{W_{h}^{\ell, t}(D)}=\left(\sum_{i=0}^{\ell}|v|_{W_{h}^{i, t}(D)}^{t}\right)^{1 / t},|v|_{W_{h}^{i, t}(D)}=\left(\sum_{K \in J_{h}}|v|_{W^{i, t}(K \cap D)}^{t}\right)^{1 / t} .
$$

Again, we write $H_{h}^{\ell}(D)=W_{h}^{\ell, 2}(D)$.

For any $v \in L^{2}(\Omega)$, we define the average and jump operators as follows: On any $e \in \Gamma_{h}$, let

$$
\begin{gathered}
\{v\}= \begin{cases}\frac{1}{2}\left(\left.v\right|_{K}+\left.v\right|_{K^{\prime}}\right) & \text { if } e \in \Gamma_{h}^{0} \text { and } e \subset \partial K \cap \partial K^{\prime}, \\
\left.v\right|_{K} & \text { if } e \in \Gamma_{h} \backslash \Gamma_{h}^{0} \text { and } e \in \partial K,\end{cases} \\
{[v]= \begin{cases}\left.v\right|_{K} n_{K}+\left.v\right|_{K^{\prime}} n_{K^{\prime}} & \text { if } e \in \Gamma_{h}^{0} \text { and } e \subset \partial K \cap \partial K^{\prime}, \\
\left.v\right|_{K} n_{K} & \text { if } e \in \Gamma_{h} \backslash \Gamma_{h}^{0} \text { and } e \in \partial K .\end{cases} }
\end{gathered}
$$


The jump and average operators can be similarly defined for vectors. It is clear that $[v]$ is a vector if $v$ is a scalar and $[v]$ is a scalar if $v$ is a vector.

We now proceed with the derivation of the mixed weak formulations of the problem (2.1) using the discontinuous test functions. To this end we rewrite the equation as a system of first-order equations. Thus, we introduce $\mathbf{u}=\nabla p$ and obtain the equations

$$
\mathbf{u}=\nabla p, \quad-\nabla \cdot \mathbf{u}=f \quad \text { in } \Omega .
$$

Multiplying the first equation of (2.2) by a function $\mathbf{v} \in H_{h}^{1}(\Omega)^{N}$ and the second equation by a $q \in H_{h}^{1}(\Omega)$, integrating by parts on each element $K \in \mathcal{J}_{h}$ and summing up over all elements, we obtain

$$
\begin{gathered}
\int_{\Omega} \mathbf{u v} d x+\sum_{K \in \mathcal{J}_{h}} \int_{K} p \nabla \cdot \mathbf{v} d x-\sum_{K \in \mathcal{J}_{h}} \int_{\partial K} p \mathbf{v} \cdot n_{K} d s=0, \\
\sum_{K \in \mathcal{J}_{h}} \int_{K} \mathbf{u} \cdot \nabla q d x-\sum_{K \in \mathcal{J}_{h}} \int_{\partial K} q \mathbf{u} \cdot n_{K} d s=\int_{\Omega} f q d x .
\end{gathered}
$$

Using the following identity for any $q \in H_{h}^{1}(\Omega), \mathbf{v} \in H_{h}^{1}(\Omega)^{N}$

$$
\sum_{K \in \mathcal{J}_{h}} \int_{\partial K} \mathbf{v} \cdot n_{K} q d s=\sum_{e \in \Gamma_{h}} \int_{e}[q]\{\mathbf{v}\} d s+\sum_{e \in \Gamma_{h}^{0}} \int_{e}\{q\}[\mathbf{v}] d s,
$$

the continuities of the solution $p$ and $\mathbf{u}$ and homogeneous boundary condition, we have

$$
\begin{gathered}
\int_{\Omega} \mathbf{u v} d x+\sum_{K \in \mathcal{J}_{h}} \int_{K} p \nabla \cdot \mathbf{v} d x-\sum_{e \in \Gamma_{h}^{0}} \int_{e}\{p\}[\mathbf{v}] d s=0, \\
\sum_{K \in \mathcal{J}_{h}} \int_{K} \mathbf{u} \cdot \nabla q d x-\sum_{e \in \Gamma_{h}} \int_{e}[q]\{\mathbf{u}\} d s=\int_{\Omega} f q d x .
\end{gathered}
$$

These are the basic weak formulas satisfied by the solution $(p, \mathbf{u})$ of the original elliptic problem. We now introduce the following bilinear forms:

$$
\begin{gathered}
a(\mathbf{u}, \mathbf{v})=\int_{\Omega} \mathbf{u v} d x, \quad c(p, q)=\sum_{e \in \Gamma_{h}} \int_{e} \lambda_{1} h_{e}^{-1}[p][q] d s \\
b(\mathbf{v}, q)=\sum_{K \in \mathcal{J}_{h}} \int_{K} q \nabla \cdot \mathbf{v} d x-\sum_{e \in \Gamma_{h}^{0}} \int_{e}\{q\}[\mathbf{v}] d s-\sum_{e \in \Gamma_{h}^{0}} \int_{e} \lambda_{2}[q][\mathbf{v}] d s,
\end{gathered}
$$

where $\lambda_{1}$ and $\lambda_{2}$ are two bounded functions and $\lambda_{1}$ is also bounded below by a positive constant. Noting that $b(\mathbf{v}, q)$ can also be rewritten as

$$
b(\mathbf{v}, q)=-\sum_{K \in \mathcal{J}_{h}} \int_{K} \mathbf{v} \cdot \nabla q d x+\sum_{e \in \Gamma_{h}} \int_{e}\{\mathbf{v}\}[q] d s-\sum_{e \in \Gamma_{h}^{0}} \int_{e} \lambda_{2}[q][\mathbf{v}] d s,
$$

we can write (2.3) and (2.4) in the following form: for any $q \in H_{h}^{2}(\Omega)$ and $\mathbf{v} \in$ $H_{h}^{2}(\Omega)^{N}$

$$
\begin{aligned}
a(\mathbf{u}, \mathbf{v})+b(\mathbf{v}, p) & =0 \\
-b(\mathbf{u}, q)+c(p, q) & =(f, q) .
\end{aligned}
$$


To define the finite element approximations, let $r \geq 1$ be a fixed integer and let $\mathbf{V}^{h} \subset H_{h}^{2}(\Omega)^{N}$ and $W^{h} \subset H_{h}^{2}(\Omega)$ be two families of finite dimensional subspaces. For simplicity, we assume that $\mathbf{V}^{h}$ is a tensor product of $W^{h}$ :

$$
\mathbf{V}^{h}=\left(W^{h}\right)^{N}, \quad W^{h}=\left\{q \in L^{\infty}(\Omega):\left.v\right|_{K} \in \mathcal{S}(K), K \in \mathcal{J}_{h}\right\},
$$

where $\mathcal{P}_{r}(K) \subset \mathcal{S}(K) \subset \mathcal{P}_{r_{1}}(K)$ and $r \leq r_{1}, \mathcal{P}_{r}(K)$ denotes the set of all polynomials of degree less than or equal to $r$. In the local discontinuous Galerkin method, the finite element approximation $\left(\mathbf{u}_{h}, p_{h}\right) \in \mathbf{V}^{h} \times W^{h}$ of $(\mathbf{u}, p)$ is sought to satisfy

$$
\begin{aligned}
a\left(\mathbf{u}_{h}, \mathbf{v}\right)+b\left(\mathbf{v}, p_{h}\right) & =0, \\
-b\left(\mathbf{u}_{h}, q\right)+c\left(p_{h}, q\right) & =(f, q)
\end{aligned}
$$

for any $q \in H_{h}^{2}(\Omega)$ and $\mathbf{v} \in H_{h}^{2}(\Omega)^{N}$. Therefore, we have the following error equation: For any $q \in W^{h}$ and $\mathbf{v} \in \mathbf{V}^{h}$, it holds that

$$
\begin{aligned}
a\left(\mathbf{u}-\mathbf{u}_{h}, \mathbf{v}\right)+b\left(\mathbf{v}, p-p_{h}\right) & =0, \\
-b\left(\mathbf{u}-\mathbf{u}_{h}, q\right)+c\left(p-p_{h}, q\right) & =0 .
\end{aligned}
$$

We shall need some special norms. For any $D \subset \Omega$, define

$$
\begin{gathered}
\|\mathbf{v}\|_{a, D}^{2}=\|\mathbf{v}\|_{L^{2}(D)}^{2}+\sum_{e \in \Gamma_{h}^{0}} \int_{e \cap D} h_{e}|[\mathbf{v}]|^{2} d s \\
\|\mathbf{v}\|_{a, 1, D}=\|\mathbf{v}\|_{L^{1}(D)}+\sum_{e \in \Gamma_{h}^{0}} \int_{e \cap D} h_{e}|[\mathbf{v}]| d s \\
|q|_{c, D}^{2}=\sum_{e \in \Gamma_{h}} \int_{e \cap D} h_{e}^{-1}|[q]|^{2} d s, \quad|q|_{c, 1, D}=\sum_{e \in \Gamma_{h}} \int_{e \cap D}|[q]| d s .
\end{gathered}
$$

Moreover, $\left|\|\cdot \mid\|_{L^{2}(D)}\right.$ denotes a modified $L^{2}$ norm:

$$
\|q\|_{L^{2}(D)}^{2}=\|q\|_{L^{2}(D)}^{2}+h^{2}\|q\|_{H_{h}^{1}(D)}^{2} .
$$

As a result of a trace theorem, the inequality

$$
|q|_{c, D} \leq C h^{-1}\|q\| \|_{L^{2}\left(D_{1}\right)}, \quad \forall q \in H_{h}^{1}\left(D_{1}\right),
$$

holds, where $D \subset D_{1}$ satisfies $\operatorname{dist}\left(D, \partial D_{1} \backslash \partial \Omega\right)>\kappa h$ for some $\kappa>0$ (see also Lemma 3.5 in Chen [10]).

Additionally, for the derivation of the pointwise error estimates we also need some weighted norms. Following Schatz [22], we introduce the weight function

$$
\sigma_{z, h}^{s}(x)=\left(\frac{h}{|x-z|+h}\right)^{s}, \quad x, z \in \Omega,-\infty<s<\infty .
$$

For $1 \leq t \leq \infty$, define

$$
\begin{gathered}
\|q\|_{L^{t}(D), z, s}=\left\|\sigma_{z, h}^{s} q\right\|_{L^{t}(D)}, \\
\|q\|_{W_{h}^{1, t}(D), z, s}=\|q\|_{L^{t}(D), z, s}+\sum_{K \mathcal{J}_{h}}\|\nabla q\|_{L^{t}(K \cap D), z, s}, \\
\|\mathbf{v}\|_{a, 1, D, z, s}=\|\mathbf{v}\|_{L^{1}(D), z, s}+\sum_{e \in \Gamma_{h}^{0}} \int_{e \cap D} h_{e} \sigma_{z, h}^{s}|[\mathbf{v}]| d s, \\
|q|_{c, 1, D, z, s}=\left|\sigma_{z, h}^{s} q\right|_{c, 1, D} .
\end{gathered}
$$

We note that although some of the norms are defined for scalar functions, they also apply to vector-valued functions in an obvious way. 
In this paper, the notation for the $L^{2}$ projections into spaces $W^{h}$ and $\mathbf{V}^{h}$ will be denoted by $Q^{h}$ the $\Pi^{h}$, respectively. More precisely, $\Pi^{h}: L^{2}(\Omega)^{N} \rightarrow \mathbf{V}^{h}$ and $Q^{h}: L^{2}(\Omega) \rightarrow W^{h}$ satisfy the equations

$$
\begin{gathered}
\left(Q^{h} q, \chi\right)=(q, \chi), \quad \forall q \in L^{2}(\Omega) \text { and } \chi \in W^{h}, \\
\left(\Pi^{h} \mathbf{v}, \psi\right)=(\mathbf{v}, \psi), \quad \forall \mathbf{v} \in L^{2}(\Omega)^{N} \text { and } \psi \in \mathbf{V}^{h} .
\end{gathered}
$$

Because of the discontinuity of functions in $\mathbf{V}^{h}$ and $W^{h}$, the operators $\Pi^{h}$ and $Q^{h}$ are eventually defined elementwise.

Before we end this section, we shall collect some known results about the approximation properties of the finite element spaces, global and local error estimates in the $L^{2}$ norm for the finite element solutions.

The first lemma below collects the standard approximation properties of the finite element spaces. These results can be easily derived by using the property of $L^{2}$ projection and the approximation properties of the finite element spaces.

Lemma 2.1. Let $0 \leq i \leq j \leq 1+r$. Then we have the following approximation properties:

(i) For any $K \in \mathcal{J}_{h}$ and $\mathbf{v} \in H^{j}(K)^{N}, q \in H^{j}(K)$, it holds that

$$
\begin{aligned}
\left\|\mathbf{v}-\Pi^{h} \mathbf{v}\right\|_{H^{i}(K)} & \leq C h^{j-i}\|\mathbf{v}\|_{H^{j}(K)}, \\
\left\|q-Q^{h} q\right\|_{H^{i}(K)} & \leq C h^{j-i}\|q\|_{H^{j}(K)} .
\end{aligned}
$$

(ii) If $D_{0} \subset D_{1} \subset \Omega$ with $\operatorname{dist}\left(D_{0}, \partial D_{1} \backslash \partial \Omega\right) \geq \kappa h$ for some $\kappa>0$, then for $\mathbf{v} \in H^{j}(K)^{N}, q \in H^{j}(K)$, it holds that

$$
\begin{aligned}
\left\|\mathbf{v}-\Pi^{h} \mathbf{v}\right\|_{H^{i}\left(D_{0}\right)} & \leq C h^{j-i}\|\mathbf{v}\|_{H^{j}\left(D_{1}\right)}, \\
\left\|q-Q^{h} q\right\|_{H^{i}\left(D_{0}\right)} & \leq C h^{j-i}\|q\|_{H^{j}\left(D_{1}\right)} .
\end{aligned}
$$

In the next lemma, we state the error estimates in the $L^{2}$ norm which can be found in Castillo, et al. 9]. The error estimate in the $L^{2}$ norm is optimal for the scalar approximation but is only sub-optimal for the vector approximation because the optimal order of approximation in $\mathbf{V}^{h}$ is $1+r$. However, it does not seem we can improve this as the numerical experiments in [9] indicate.

Lemma 2.2. Let $(p, \mathbf{u})$ and $\left(p_{h}, \mathbf{u}_{h}\right)$ satisfy 2.10) and $(p, \mathbf{u}) \in H^{r+1}(\Omega) \times H^{r}(\Omega)^{N}$. Then we have

$$
\left\|p-p_{h}\right\|_{L^{2}(\Omega)}+h\left|p-p_{h}\right|_{c, \Omega}+h\left\|\mathbf{u}-\mathbf{u}_{h}\right\|_{a, \Omega} \leq C h^{1+r}\|p\|_{H^{1+r}(\Omega)} .
$$

The results in the following lemma, which are based on the local error estimates obtained in Chen 10, are crucial to the proof of the pointwise error estimates.

Lemma 2.3. Let $\Omega_{0} \subset \Omega_{1} \subset \Omega$ be subdomains with $d=\operatorname{dist}\left(\Omega_{0}, \partial \Omega_{1} \backslash \partial \Omega\right) \geq M h$ for sufficiently large $M>1$ and let $\partial \Omega_{1} \cap \partial \Omega$ be sufficiently smooth. Let $(p, \mathbf{u})$ and $\left(p_{h}, \mathbf{u}_{h}\right)$ satisfy (2.10) and $(p, \mathbf{u}) \in H^{r+1}\left(\Omega_{1}\right) \times H^{r}\left(\Omega_{1}\right)^{N}$, and let $t \geq 0$ and $t_{1}=0,1$. Then we have

$$
\begin{aligned}
\| p- & p_{h}\left\|_{L^{2}\left(\Omega_{0}\right)} \leq C h^{1+r}\right\| p \|_{H^{1+r}\left(\Omega_{1}\right)} \\
& +C d^{-N / 2-t}\left(\left\|p-p_{h}\right\|_{W^{-t, 1}\left(\Omega_{1}\right)}+d^{1-t_{1}}\left\|\mathbf{u}-\mathbf{u}_{h}\right\|_{W^{-t-t_{1}, 1}\left(\Omega_{1}\right)}\right)
\end{aligned}
$$


and for any fixed $\varepsilon \in\left(0, \frac{1}{2}\right)$

$$
\begin{aligned}
& \left\|\mathbf{u}-\mathbf{u}_{h}\right\|_{a, \Omega_{0}}+\left|p-p_{h}\right|_{c, \Omega_{0}} \leq C h^{r}\|p\|_{H^{1+r}\left(\Omega_{1}\right)} \\
& \quad+C d^{-N / 2-t-1}\left(\frac{h}{d}\right)^{-\varepsilon}\left(\left\|p-p_{h}\right\|_{W^{-t, 1}\left(\Omega_{1}\right)}+d^{1-t_{1}}\left\|\mathbf{u}-\mathbf{u}_{h}\right\|_{W^{-t-t_{1}, 1}\left(\Omega_{1}\right)}\right) .
\end{aligned}
$$

Here the positive constant $C$ in (2.16) depends on $\varepsilon$.

Proof. Without loss of generality, we assume that $\Omega$ is the unit ball in $R^{N}$. It suffices to show Lemma 2.3 with $\Omega_{0}$ and $\Omega_{1}$ being the spheres of radii $d / 2$ and $d$, respectively, with centers at $x=0$. Assume that $x$ denotes the variable on $\Omega$. Let $\tilde{x}=x / d$ be the new variable on the transferred regions $\tilde{\Omega}_{0}$ and $\tilde{\Omega}_{1}$. Then $\operatorname{dist}\left(\tilde{\Omega}_{0}, \partial \tilde{\Omega}_{1}\right)=1 / 2$. Set

$$
\tilde{p}(\tilde{x})=\frac{p(\tilde{x} d)}{d}, \quad \tilde{\mathbf{u}}(\tilde{x})=\mathbf{u}(\tilde{x} d), \quad \tilde{p_{h}}=\frac{p_{h}(\tilde{x} d)}{d}, \quad \tilde{\mathbf{u}}_{h}=\mathbf{u}_{h}(\tilde{x} d), \quad \tilde{f}(\tilde{x})=f(\tilde{x} d) .
$$

Then we have for any $\tilde{q} \in H_{h}^{2}(\tilde{\Omega})$ and $\tilde{\mathbf{v}} \in H_{h}^{2}(\tilde{\Omega})^{N}$

$$
\begin{aligned}
\tilde{a}(\tilde{\mathbf{u}}, \tilde{\mathbf{v}}) & +\tilde{b}(\tilde{\mathbf{v}}, \tilde{p})=0, \\
-\tilde{b}(\tilde{\mathbf{u}}, \tilde{q})+\tilde{c}(\tilde{p}, \tilde{q}) & =(\tilde{f}, \tilde{q})
\end{aligned}
$$

and for any $\tilde{q}_{h} \in \tilde{W}^{h}$ and $\tilde{\mathbf{v}}_{h} \in \tilde{\mathbf{V}}^{h}$

$$
\begin{aligned}
\tilde{a}\left(\tilde{\mathbf{u}}_{h}, \tilde{\mathbf{v}}_{h}\right) & +\tilde{b}\left(\tilde{\mathbf{v}}_{h}, \tilde{p}_{h}\right)=0, \\
-\tilde{b}\left(\tilde{\mathbf{u}}_{h}, \tilde{q}_{h}\right)+\tilde{c}\left(\tilde{p}_{h}, \tilde{q}_{h}\right) & =\left(\tilde{f}, \tilde{q}_{h}\right) .
\end{aligned}
$$

Here

$$
\begin{gathered}
\tilde{a}(\tilde{\mathbf{u}}, \tilde{\mathbf{v}})=\int_{\tilde{\Omega}} \tilde{\mathbf{u}} \tilde{\mathbf{v}} d \tilde{x}, \quad \tilde{c}(\tilde{p}, \tilde{q})=\sum_{\tilde{e} \in \tilde{\Gamma}_{h}} \int_{\tilde{e}} \lambda_{1}\left(h_{e} / d\right)^{-1}[\tilde{p}][\tilde{q}] d s . \\
\tilde{b}(\tilde{\mathbf{v}}, \tilde{q})=\sum_{\tilde{K} \in \tilde{J}_{h}} \int_{\tilde{K}} \tilde{q} \nabla \cdot \tilde{\mathbf{v}} d \tilde{x}-\sum_{\tilde{e} \in \tilde{\Gamma}_{h}^{0}} \int_{\tilde{e}}\{\tilde{q}\}[\tilde{\mathbf{v}}] d s-\sum_{\tilde{e} \in \tilde{\Gamma}_{h}^{0}} \int_{\tilde{e}} \lambda_{2}[\tilde{q}][\tilde{\mathbf{v}}] d s .
\end{gathered}
$$

Applying the results of Theorem 4.1 in Chen [10] for $\tilde{p}-\tilde{p}_{h}$, we have

$$
\begin{aligned}
\| \tilde{p}- & \tilde{p}_{h} \|_{L^{2}\left(\tilde{\Omega}_{0}\right)} \leq C\left(\frac{h}{d}\right)^{1+r}|\tilde{p}|_{H^{1+r}\left(\tilde{\Omega}_{1}\right)} \\
& +C\left(\left\|\tilde{p}-\tilde{p}_{h}\right\|_{W^{-t, 1}(\tilde{\Omega})}+\left\|\tilde{\mathbf{u}}-\tilde{\mathbf{u}}_{h}\right\|_{W^{-t-t_{1}, 1}(\tilde{\Omega})}\right) .
\end{aligned}
$$

Changing the variable $\tilde{x}$ back to the original variable $x$ in (2.21) gives (2.15). Likewise, the estimate (2.16) can be proved in a similar way.

\section{Pointwise error estimate}

The main result of this section is the pointwise error estimate for the scalar approximation $p_{h}$ which is stated in Theorem 3.1. The proof of the main result is based on a series of lemmas provided in the section.

Theorem 3.1. Let $(p, \mathbf{u})$ and $\left(p_{h}, \mathbf{u}_{h}\right)$ satisfy (2.10) and $0 \leq s \leq r-1$. Then there is a constant $C>0$ such that for any $z \in \bar{\Omega}$, we have

$$
\left|\left(p-p_{h}\right)(z)\right| \leq C|\ln h|^{\bar{s}}\left(\left\|p-Q^{h} p\right\|_{L^{\infty}(\Omega), z, s}+h\left\|\mathbf{u}-\Pi^{h} \mathbf{u}\right\|_{L^{\infty}(\Omega), z, s}\right),
$$

where $\bar{s}=0$ if $0 \leq s<r-1$ and $\bar{s}=1$ if $s=r-1$. 
Proof. Let $K_{z} \in \mathcal{J}_{h}$ be such that $z \in \bar{K}_{z}$. Construct a function $\delta_{z} \in C_{0}^{1}\left(\bar{K}_{z}\right)$. Namely, $\delta_{z}$ and its partial derivatives are continuous in $\Omega$ and have a compact support in the closure of $K_{z}$. In addition to this, we require that function $\delta_{z}$ satisfies the following properties:

$$
\left(\delta_{z}, q_{h}\right)=q_{h}(z), \quad \forall q_{h} \in W^{h},
$$

and

$$
\left\|\delta_{z}\right\|_{L^{t}(\Omega)}+h\left\|\delta_{z}\right\|_{W^{1, t}(\Omega)} \leq C h^{-N / t^{\prime}}, \quad 1 \leq t \leq \infty, \quad \frac{1}{t}+\frac{1}{t^{\prime}}=1 .
$$

We point out that the requirements on the derivatives of $\delta_{z}$ are not used in this proof but in the proof of Theorem 4.1. By the triangle inequality,

$$
\begin{aligned}
\left|\left(p-p_{h}\right)(z)\right| & \leq\left|\left(p-Q^{h} p\right)(z)\right|+\left|\left(\delta_{z}, Q^{h} p-p_{h}\right)\right| \\
& \leq\left|\left(p-Q^{h} p\right)(z)\right|+\left|\left(\delta_{z}, Q^{h} p-p\right)\right|+\left|\left(\delta_{z}, p-p_{h}\right)\right| \\
& \leq C\left\|p-Q^{h} p\right\|_{L^{\infty}(\Omega), z, s}+\left|\left(\delta_{z}, p-p_{h}\right)\right| .
\end{aligned}
$$

Let $g_{z} \in H_{0}^{1}(\Omega)$ be the solution of the elliptic problem

$$
-\Delta g_{z}=\delta_{z}
$$

and $\mathbf{G}_{z}=\nabla g_{z}$. We may call $g_{z}$ a regularized Green's function. Furthermore, let $\left(g_{z, h}, \mathbf{G}_{z, h}\right) \in W^{h} \times \mathbf{V}^{h}$ be the finite element approximation of $\left(g_{z}, \mathbf{G}_{z}\right)$ satisfying

$$
\begin{aligned}
a\left(\mathbf{G}_{z}-\mathbf{G}_{z, h}, \mathbf{v}\right)+b\left(\mathbf{v}, g_{z}-g_{z, h}\right) & =0, \\
-b\left(\mathbf{G}_{z}-\mathbf{G}_{z, h}, q\right) & +c\left(g_{z}-g_{z, h}, q\right)=0
\end{aligned}
$$

for any $(q, \mathbf{v}) \in W^{h} \times \mathbf{V}^{h}$. Then a simple manipulation leads to

$$
\begin{aligned}
\left(\delta_{z}, p-p_{h}\right)= & -b\left(\mathbf{G}_{z}, p-p_{h}\right)+c\left(g_{z}, p-p_{h}\right) \\
= & a\left(\mathbf{G}_{z, h}-\mathbf{G}_{z}, \mathbf{u}-\Pi^{h} \mathbf{u}\right)+b\left(\mathbf{u}-\Pi^{h} \mathbf{u}, g_{z, h}-g_{z}\right) \\
& +b\left(\mathbf{G}_{z}-\mathbf{G}_{z, h}, Q^{h} p-p\right)+c\left(g_{z},-g_{z, h}, p-Q^{h} p\right) .
\end{aligned}
$$

By the Cauchy-Schwarz inequality and the definitions of the related norms, the first and the last terms in (3.4) can be bounded as follows:

$$
\begin{aligned}
a\left(\mathbf{G}_{z, h}-\mathbf{G}_{z}, \mathbf{u}-\Pi^{h} \mathbf{u}\right)+c\left(g_{z}-g_{z, h}, p-Q^{h} p\right) \\
\leq \quad C\left\|\mathbf{u}-\Pi^{h} \mathbf{u}\right\|_{L^{\infty}(\Omega), z, s}\left\|\mathbf{G}_{z}-\mathbf{G}_{z, h}\right\|_{L^{1}(\Omega), z,-s} \\
\quad+C h^{-1}\left\|p-Q^{h} p\right\|_{L^{\infty}(\Omega), z, s}\left|g_{z}-g_{z, h}\right|_{c, 1, \Omega, z,-s} .
\end{aligned}
$$

Next, we shall deal with the second and third terms on the right-hand side of (3.4). According to (2.7), (2.6) and the orthogonal properties of the operators $Q^{h}$ and $\Pi^{h}$, we have

$$
\begin{aligned}
b(\mathbf{u}- & \left.\Pi^{h} \mathbf{u}, g_{z}-g_{z, h}\right)=-\sum_{K \in \mathcal{J}_{h}} \int_{K}\left(\mathbf{u}-\Pi^{h} \mathbf{u}\right) \nabla\left(g_{z}-Q^{h} g_{z}\right) d x \\
& +\sum_{e \in \Gamma_{h}} \int_{e}\left\{\mathbf{u}-\Pi^{h} \mathbf{u}\right\}\left[g_{z}-g_{z, h}\right] d s-\sum_{e \in \Gamma_{h}^{0}} \int_{e} \lambda_{2}\left[g_{z}-g_{z, h}\right]\left[\mathbf{u}-\Pi_{h} \mathbf{u}\right] d s
\end{aligned}
$$

and

$$
\begin{aligned}
& b\left(\mathbf{G}_{z}-\mathbf{G}_{z, h}, Q^{h} p-p\right)=\sum_{K \in \mathcal{J}_{h}} \int_{K}\left(p-Q^{h} p\right) \nabla \cdot\left(\mathbf{G}_{z}-\Pi^{h} \mathbf{G}_{z}\right) d x \\
& -\sum_{e \in \Gamma_{h}} \int_{e}\left\{p-Q^{h} p\right\}\left[\mathbf{G}_{z}-\mathbf{G}_{z, h}\right] d s-\sum_{e \in \Gamma_{h}^{0}} \int_{e} \lambda_{2}\left[p-Q^{h} p\right]\left[\mathbf{G}_{z}-\mathbf{G}_{z, h}\right] d s .
\end{aligned}
$$


As a result of using the Cauchy-Schwarz inequality for the six integrals on the right-hand side of (3.6) and (3.7), one has

$$
\begin{aligned}
& b\left(\mathbf{u}-\Pi^{h} \mathbf{u}, g_{z}-g_{z, h}\right) \\
\leq & C\left\|\mathbf{u}-\Pi^{h} \mathbf{u}\right\|_{L^{\infty}(\Omega), z, s}\left(\left\|\nabla\left(g_{z}-Q^{h} g_{z}\right)\right\|_{L^{1}(\Omega), z,-s}+\left|g_{z}-g_{z, h}\right|_{c, 1, \Omega, z,-s}\right)
\end{aligned}
$$

and

$$
\begin{aligned}
& b\left(\mathbf{G}_{z}-\Pi^{h} \mathbf{G}_{z}, Q^{h} p-p\right) \leq C h^{-1}\left\|p-Q^{h} p\right\|_{L^{\infty}(\Omega), z, s} \\
& \quad \cdot\left(h\left|\nabla \cdot\left(\mathbf{G}_{z}-\Pi^{h} \mathbf{G}_{z}\right)\right|_{L^{1}(\Omega), z,-s}+\left\|\mathbf{G}_{z}-\mathbf{G}_{z, h}\right\|_{a, 1, \Omega, z,-s}\right) .
\end{aligned}
$$

Consequently, inserting (3.5), (3.8) and (3.9) in (3.4) results in

$$
\begin{aligned}
\left|\left(\delta_{z}, p-p_{h}\right)\right| & \\
\leq & \left(h^{-1}\left\|p-Q^{h} p\right\|_{L^{\infty}(\Omega), z, s}+\left\|\mathbf{u}-\Pi^{h} \mathbf{u}\right\|_{L^{\infty}(\Omega), z, s}\right) \\
& \cdot\left(\left\|\nabla\left(g_{z}-Q^{h} g_{z}\right)\right\|_{L^{1}(\Omega), z,-s}+h\left\|\nabla \cdot\left(\mathbf{G}_{z}-\Pi^{h} \mathbf{G}_{z}\right)\right\|_{L^{1}(\Omega), z,-s}\right. \\
& \left.+\left|g_{z}-g_{z, h}\right|_{c, 1, \Omega, z,-s}+\left\|\mathbf{G}_{z}-\mathbf{G}_{z, h}\right\|_{a, 1, \Omega, z,-s}\right) .
\end{aligned}
$$

Applying the estimates contained in Lemmas 3.2 and 3.4 into the above inequality and then inserting the resulting estimate into (3.1), we deduce the desired estimate of the theorem. The proof is complete.

The rest of this section is devoted to providing error estimates for $g_{z}-g_{z, h}$ and $\mathbf{G}_{z}-\mathbf{G}_{z, h}$ through a number of lemmas. Without loss of generality we assume $\operatorname{diam}(\Omega) \leq 1$ and define

$$
d_{j}=2^{-j} \quad \text { for } j=0,1,2, \ldots,
$$

and for any fixed $z \in \bar{\Omega}$, set

$$
\begin{aligned}
\Omega_{j} & =\left\{x \in \Omega: \quad d_{j+1}<|x-z|<d_{j}\right\}, \\
\Omega_{j}^{(1)} & =\left\{x \in \Omega: d_{j+2}<|x-z|<d_{j-1}\right\}, \\
\Omega_{j}^{(2)} & =\left\{x \in \Omega: d_{j+3}<|x-z|<d_{j-2}\right\}, \\
\Omega_{j}^{(3)} & =\left\{x \in \Omega: d_{j+4}<|x-z|<d_{j-3}\right\} .
\end{aligned}
$$

We start with the following result about an auxiliary problem used in the proof Lemma 3.4

Lemma 3.1. For $\varphi \in C_{0}^{\infty}\left(\Omega_{j}^{(1)}\right)$ satisfying $\|\varphi\|_{L^{t}(\Omega)} \leq 1$, let $w \in H_{0}^{1}(\Omega)$ be the solution of $-\Delta w=\varphi$ in $\Omega$. Then we have

$$
\|w\|_{W^{1+r, \infty}\left(\Omega \backslash \Omega_{j}^{(2)}\right)} \leq C d_{j}^{1-r-N / t}, \quad 1 \leq t \leq \infty .
$$

Proof. For any $x \in \Omega \backslash \Omega_{j}^{(2)}$, let $G_{x}$ denote the Green's function of problem (2.1) with singularity at $x$. Then we have (see Agmon, Douglis and Nirenberg [1])

$$
w(x)=\int_{\Omega} G_{x}(y) \varphi(y) d y
$$

and

$$
\left|\frac{\partial^{\alpha+\beta} G_{x}(y)}{\partial x^{\alpha} \partial y^{\beta}}\right| \leq C|x-y|^{2-N-|\alpha|-|\beta|} \quad \text { for } \quad|\alpha|+|\beta|>0
$$


Differentiating (3.13) with respect to $x$, for $x \in \Omega \backslash \Omega_{j}^{(2)}$ and $|\alpha| \leq 1+r$ we have

$$
\begin{aligned}
\left|\frac{\partial^{\alpha} w(x)}{\partial x^{\alpha}}\right| & =\left|\int_{\Omega} \frac{\partial^{\alpha} G_{x}(y)}{\partial x^{\alpha}} \varphi(y) d y\right| \\
& \leq C \int_{\Omega_{j}^{(1)}}|x-y|^{2-N-|\alpha|}|\varphi(y)| d y \\
& \leq C d_{j}^{1-N-r} d_{j}^{N(1-1 / t)}\|\varphi\|_{L^{t}\left(\Omega_{j}^{(1)}\right)} \leq C d_{j}^{1-r-N / t} .
\end{aligned}
$$

This completes the proof.

In the next lemma, we show a bound for the derivatives of the "regularized Green's function" $g_{z}$ in the regions away from its singularity and an error estimate for the $L^{2}$ projection of $g_{z}$ in the weighted $W^{1,1}$ norm.

Lemma 3.2. Let $g_{z} \in H_{0}^{1}(\Omega)$ be the solution of (3.2). Then we have

$$
\left\|g_{z}\right\|_{H^{1+r}\left(\Omega_{j}^{(1)}\right)} \leq C d_{j}^{1-r-N / 2}
$$

and

$$
\left\|\nabla\left(g_{z}-Q^{h} g_{z}\right)\right\|_{L^{1}(\Omega), z,-s}+h\left\|\nabla \cdot\left(\mathbf{G}_{z}-\Pi^{h} \mathbf{G}_{z}\right)\right\|_{L^{1}(\Omega), z,-s} \leq C h|\ln h|^{\bar{s}},
$$

where $\mathbf{G}_{z}=\nabla g_{z}, \bar{s}=1$ if $0 \leq s<r-1$ and $\bar{s}=1$ if $s=r-1$.

Proof. For any $x \in \Omega_{j}^{(1)}$, let $G_{x}$ be Green's function of problem (2.1) with singularity at $x$. Then we have

$$
g_{z}(x)=\int_{\Omega} G_{x}(y) \delta_{z}(y) d y
$$

and $G_{x}(y)$ satisfies the inequality (3.14). Differentiating (3.17) with respect to $x$, we have for $x \in \Omega_{j}^{(1)}$ and $|\alpha| \leq 1+r$

$$
\begin{aligned}
\left|\frac{\partial^{\alpha} g(x)}{\partial x^{\alpha}}\right| & =\left|\int_{\Omega} \frac{\partial^{\alpha} G_{x}(y)}{\partial x^{\alpha}} \delta_{z}(y) d y\right| \\
& \leq C \int_{K_{z}}|x-y|^{2-N-|\alpha|}\left|\delta_{z}(y)\right| d y \\
& \leq C d_{j}^{1-N-r}\left\|\delta_{z}\right\|_{L^{1}\left(K_{z}\right)} \leq C d_{j}^{1-N-r} .
\end{aligned}
$$

Integrating (3.18) over $\Omega_{j}^{(1)}$ gives us the desired result (3.15). We now show (3.16). By the triangle and the Cauchy-Schwarz inequalities, we have

$$
\begin{aligned}
& \left\|\nabla\left(g_{z}-Q^{h} g_{z}\right)\right\|_{L^{1}(\Omega), z,-s} \\
& \leq\left\|\nabla\left(g_{z}-Q^{h} g_{z}\right)\right\|_{L^{1}\left(B_{M h}(z)\right), z,-s}+\sum_{j=0}^{J}\left\|\nabla\left(g_{z}-Q^{h} g_{z}\right)\right\|_{L^{1}\left(\Omega_{j}\right), z,-s} \\
& \leq C h^{N / 2+1}\left\|g_{z}\right\|_{H^{2}(\Omega)}+C \sum_{j=0}^{J} d_{j}^{N / 2+s} h^{r-s}\left\|g_{z}\right\|_{H^{1+r}\left(\Omega_{j}^{(1)}\right)} .
\end{aligned}
$$

Using the $H^{2}$ a priori regularity $\left\|g_{z}\right\|_{H^{2}(\Omega)} \leq C\left\|\delta_{z}\right\|_{L^{2}\left(K_{z}\right)} \leq C h^{-N / 2}$ and the estimate (3.15) in (3.19), we have

$$
\left\|\nabla\left(g_{z}-Q^{h} g_{z}\right)\right\|_{L^{1}(\Omega), z,-s} \leq C h+C \Theta(r-1-s) h \leq C h|\ln h|^{\bar{s}} .
$$


Here the function $\Theta(\gamma)$ is defined by

$$
\Theta(\gamma)=\sum_{j=0}^{J}\left(\frac{h}{d_{j}}\right)^{\gamma} \leq C \begin{cases}\ln \frac{1}{h} & \text { if } \gamma=0, \\ \frac{1}{M^{\gamma}\left(1-2^{-\gamma}\right)} & \text { if } \gamma>0 .\end{cases}
$$

With a similar procedure, we can obtain

$$
\left\|\nabla\left(\mathbf{G}_{z}-\Pi^{h} \mathbf{G}_{z}\right)\right\|_{L^{1}(\Omega), z,-s} \leq C+C \Theta(r-s) \leq C
$$

for any $0 \leq s \leq r-1$. Thus, (3.20) and (3.22) prove the lemma.

The next lemma is used in the proof of Lemma 3.4 for $q=g_{z}, \mathbf{v}=\mathbf{G}_{z}, q_{h}=g_{z, h}$ and $\mathbf{v}_{h}=\mathbf{G}_{z, h}$. It will also be used in the next section for different $q, q_{h}, \mathbf{v}$ and $\mathbf{v}_{h}$.

Lemma 3.3. For $\varphi \in C_{0}^{\infty}\left(\Omega_{j}^{(1)}\right)$ satisfying $\|\varphi\|_{L^{\infty}(\Omega)} \leq 1$, let $w \in H_{0}^{1}(\Omega)$ be the solution of $-\Delta w=\varphi$ in $\Omega$ and $\mathbf{\Phi}=\nabla w$. Then for any $\mathbf{v}_{h} \in \mathbf{V}_{h}, q_{h} \in W^{h}$ and $\mathbf{v} \in H_{h}^{1}(\Omega)^{N}, q \in H_{h}^{1}(\Omega)$, we have

$$
\begin{aligned}
a(\mathbf{\Phi}- & \left.\Pi^{h} \mathbf{\Phi}, \mathbf{v}-\mathbf{v}_{h}\right)+b\left(\mathbf{\Phi}-\Pi^{h} \mathbf{\Phi}, q-q_{h}\right) \\
& +b\left(\mathbf{v}-\mathbf{v}_{h}, w-Q^{h} w\right)-c\left(w-Q^{h} w, q-q_{h}\right) \\
\leq & C h^{1+r} d_{j}^{N / 2}\left(\|q\|_{H^{1+r}\left(\Omega_{j}^{(3)}\right)}+\|\mathbf{v}\|_{H^{r}\left(\Omega_{j}^{(3)}\right)}\right) \\
& +C h^{r} d_{j}^{1-r}\left(\left|\nabla\left(q-Q^{h} q\right)\right|_{L^{1}(\Omega)}+h\left|\nabla \cdot\left(\mathbf{v}-\Pi^{h} \mathbf{v}\right)\right|_{L^{1}(\Omega)}\right) \\
& +C h^{r} d_{j}^{1-r}\left(\left|q-q_{h}\right|_{c, 1, \Omega}+\left\|\mathbf{v}-\mathbf{v}_{h}\right\|_{a, 1, \Omega}\right) \\
& +C h d_{j}^{N / 2}\left(\left\|\mathbf{v}-\mathbf{v}_{h}\right\|_{a, \Omega_{j}^{(2)}}+\left|q-q_{h}\right|_{c, \Omega_{j}^{(2)}}\right) .
\end{aligned}
$$

Proof. Let us consider the following decomposition:

$$
\begin{aligned}
& a\left(\boldsymbol{\Phi}-\Pi^{h} \mathbf{\Phi}, \mathbf{v}-\mathbf{v}_{h}\right)+b\left(\boldsymbol{\Phi}-\Pi^{h} \mathbf{\Phi}, q-q_{h}\right) \\
& \quad+b\left(\mathbf{v}-\mathbf{v}_{h}, w-Q^{h} w\right)-c\left(w-Q^{h} w, q-q_{h}\right)=I_{1}+I_{2},
\end{aligned}
$$

where

$$
\begin{aligned}
I_{1}= & a_{\Omega \backslash \Omega_{j}^{(2)}}\left(\mathbf{\Phi}-\Pi^{h} \mathbf{\Phi}, \mathbf{v}-\mathbf{v}_{h}\right)+b_{\Omega \backslash \Omega_{j}^{(2)}}\left(\mathbf{\Phi}-\Pi^{h} \mathbf{\Phi}, q-q_{h}\right) \\
& +b_{\Omega \backslash \Omega_{j}^{(2)}}\left(\mathbf{v}-\mathbf{v}_{h}, w-Q^{h} w\right)-c_{\Omega \backslash \Omega_{j}^{(2)}}\left(w-Q^{h} w, q-q_{h}\right), \\
I_{2}= & a_{\Omega_{j}^{(2)}}\left(\mathbf{\Phi}-\Pi^{h} \mathbf{\Phi}, \mathbf{v}-\mathbf{v}_{h}\right)+b_{\Omega_{j}^{(2)}}\left(\mathbf{\Phi}-\Pi^{h} \mathbf{\Phi}, q-q_{h}\right) \\
& +b_{\Omega_{j}^{(2)}}\left(\mathbf{v}-\mathbf{v}_{h}, w-Q^{h} w\right)-c_{\Omega_{j}^{(2)}}\left(w-Q^{h} w, q-q_{h}\right) .
\end{aligned}
$$

We shall estimate all terms of $I_{1}$ and $I_{2}$. For the first and last term of $I_{1}$, applying the Cauchy-Schwarz inequality and Lemma 3.1 with $t=\infty$, we have

$$
\begin{aligned}
& \left|a_{\Omega \backslash \Omega_{j}^{(2)}}\left(\mathbf{\Phi}-\Pi^{h} \mathbf{\Phi}, \mathbf{v}-\mathbf{v}_{h}\right)\right|+\left|c_{\Omega \backslash \Omega_{j}^{(2)}}\left(w-Q^{h} w, q-q_{h}\right)\right| \\
& \quad \leq C h^{r}\|w\|_{W^{1+r, \infty}\left(\Omega \backslash \Omega_{j}^{(1)}\right)}\left(\left\|\mathbf{v}-\mathbf{v}_{h}\right\|_{L^{1}(\Omega)}+\left|q-q_{h}\right|_{c, 1, \Omega}\right) \\
& \quad \leq C h^{r} d_{j}^{1-r}\left(\left\|\mathbf{v}-\mathbf{v}_{h}\right\|_{L^{1}(\Omega)}+\left|q-q_{h}\right|_{c, 1, \Omega}\right) .
\end{aligned}
$$


For the second term of $I_{1}$, we recall the formula (2.7) for the bilinear $b$ and note that $\nabla W^{h} \subset \mathbf{V}^{h}$. We have

$$
\begin{aligned}
b_{\Omega \backslash \Omega_{j}^{(2)}} & \left(\boldsymbol{\Phi}-\Pi^{h} \boldsymbol{\Phi}, q-q_{h}\right) \\
= & -\sum_{K \in \mathcal{J}_{h}} \int_{K \cap\left(\Omega \backslash \Omega_{j}^{(2)}\right)}\left(\boldsymbol{\Phi}-\Pi^{h} \boldsymbol{\Phi}\right) \nabla\left(q-Q^{h} q\right) d x \\
& +\sum_{e \in \Gamma_{h}} \int_{e \cap\left(\Omega \backslash \Omega_{j}^{(2)}\right)}\left\{\boldsymbol{\Phi}-\Pi^{h} \boldsymbol{\Phi}\right\}\left[q-q_{h}\right] d s \\
& -\sum_{e \in \Gamma_{h}^{0}} \int_{e \cap\left(\Omega \backslash \Omega_{j}^{(2)}\right)} \lambda_{2}\left[q-q_{h}\right]\left[\mathbf{\Phi}-\Pi_{h} \boldsymbol{\Phi}\right] d s .
\end{aligned}
$$

By the Cauchy-Schwarz inequality and Lemma 3.1 with $t=\infty$, the terms on the right-hand side of (3.26) are bounded as follows:

$$
\begin{gathered}
-\sum_{K \in \mathcal{J}_{h}} \int_{K \cap\left(\Omega \backslash \Omega_{j}^{(2)}\right)}\left(\boldsymbol{\Phi}-\Pi^{h} \mathbf{\Phi}\right) \nabla\left(q-Q^{h} q\right) d x \\
\leq \quad C h^{r}\|w\|_{W^{1+r, \infty}\left(\Omega \backslash \Omega_{j}^{(1)}\right)}\left\|\nabla\left(q-Q^{h} q\right)\right\|_{W^{1,1}\left(\Omega \backslash \Omega_{j}^{(2)}\right)} \\
\leq \quad C h^{r} d_{j}^{1-r}\left\|\nabla\left(q-Q^{h} q\right)\right\|_{L^{1}(\Omega)} \\
\quad \sum_{e \in \Gamma_{h}} \int_{e \cap\left(\Omega \backslash \Omega_{j}^{(2)}\right)}\left\{\boldsymbol{\Phi}-\Pi^{h} \mathbf{\Phi}\right\}\left[q-q_{h}\right] d s \\
\leq C h^{r}\|w\|_{W^{1+r, \infty}\left(\Omega \backslash \Omega_{j}^{(2)}\right)}\left|q-q_{h}\right|_{c, 1, \Omega \backslash \Omega_{j}^{(2)}} \\
\leq C h^{r} d_{j}^{1-r}\left|q-q_{h}\right|_{c, 1, \Omega}, \\
\sum_{e \in \Gamma_{h}^{0}} \int_{e \cap\left(\Omega \backslash \Omega_{j}^{(2)}\right)} \lambda_{2}\left[q-q_{h}\right]\left[\mathbf{\Phi}-\Pi_{h} \mathbf{\Phi}\right] d s \leq C h^{r} d_{j}^{1-r}\left|q-q_{h}\right|_{c, 1, \Omega}
\end{gathered}
$$

Hence, inserting (3.27), (3.28) and (3.29) in (3.26), we obtain the estimate for the second term of $I_{1}$ :

$$
b_{\Omega \backslash \Omega_{j}^{(2)}}\left(\mathbf{\Phi}-\Pi^{h} \mathbf{\Phi}, q-q_{h}\right) \leq C h^{r} d_{j}^{1-r}\left(\left\|\nabla\left(q-Q^{h} q\right)\right\|_{L^{1}(\Omega)}+\left|q-q_{h}\right|_{c, 1, \Omega}\right) .
$$

For the third term of $I_{1}$, we use the formula (2.6) of bilinear form $b$ to write

$$
\begin{aligned}
b_{\Omega \backslash \Omega_{j}^{(2)}} & \left(\mathbf{v}-\mathbf{v}_{h}, w-Q^{h} w\right) \\
= & -\sum_{K \in \mathcal{J}_{h}} \int_{K \cap\left(\Omega \backslash \Omega_{j}^{(2)}\right)}\left(w-Q^{h} w\right) \nabla \cdot\left(\mathbf{v}-\Pi^{h} \mathbf{v}\right) d x \\
& -\sum_{e \in \Gamma_{h}^{0}} \int_{e \cap\left(\Omega \backslash \Omega_{j}^{(2)}\right)}\left\{w-Q^{h} w\right\}\left[\mathbf{v}-\mathbf{v}_{h}\right] d s \\
& -\sum_{e \in \Gamma_{h}^{0}} \int_{e \cap\left(\Omega \backslash \Omega_{j}^{(2)}\right)} \lambda_{2}\left[w-Q^{h} w\right]\left[\mathbf{v}-\mathbf{v}_{h}\right] d s .
\end{aligned}
$$

The three term on the right-hand side of (3.31) can be estimated in the same way as those of (3.26). We deduce that

$$
b_{\Omega \backslash \Omega_{j}^{(2)}}\left(\mathbf{v}-\mathbf{v}_{h}, w-Q^{h} w\right) \leq C h^{r} d_{j}^{1-r}\left(h\left\|\nabla \cdot\left(\mathbf{v}-\Pi^{h} \mathbf{v}\right)\right\|_{L^{1}(\Omega)}+\left\|\mathbf{v}-\mathbf{v}_{h}\right\|_{a, 1, \Omega}\right) .
$$


On combining (3.25), (3.30) and (3.32), we obtain the estimate for $I_{1}$ :

$$
\begin{aligned}
I_{1} \leq & C h^{r} d_{j}^{1-r}\left(\left\|\nabla\left(q-Q^{h} q\right)\right\|_{L^{1}(\Omega)}+h\left\|\nabla \cdot\left(\mathbf{v}-\Pi^{h} \mathbf{v}\right)\right\|_{L^{1}(\Omega)}\right) \\
& +C h^{r} d_{j}^{1-r}\left(\left|q-q_{h}\right|_{c, 1, \Omega}+\left\|\mathbf{v}-\mathbf{v}_{h}\right\|_{a, 1, \Omega}\right) .
\end{aligned}
$$

It remains to estimate terms of $I_{2}$. Like $I_{1}$, we shall first estimate the first and the last terms of $I_{2}$ and then the second and third terms of $I_{2}$. In fact, for the first and last terms of $I_{2}$, using the inequality (2.14) and the approximation property of $Q^{h}$ to get

$$
\left|w-Q^{h} w\right|_{c, \Omega_{j}^{(2)}} \leq C h\|w\|_{H^{2}\left(\Omega_{j}^{(3)}\right)}
$$

and applying the Cauchy-Schwarz inequality, we have

$$
\begin{aligned}
& \left|a_{\Omega_{j}^{(2)}}\left(\boldsymbol{\Phi}-\Pi^{h} \mathbf{\Phi}, \mathbf{v}-\mathbf{v}_{h}\right)\right|+\left|c_{\Omega_{j}^{(2)}}\left(w-Q^{h} w, q-q_{h}\right)\right| \\
& \quad \leq C h\|w\|_{H^{2}\left(\Omega_{j}^{(3)}\right)}\left(\left\|\mathbf{v}-\mathbf{v}_{h}\right\|_{L^{2}\left(\Omega_{j}^{(2)}\right)}+\left|q-q_{h}\right|_{c, \Omega_{j}^{(2)}}\right) \\
& \quad \leq C h\|\varphi\|_{L^{2}\left(\Omega_{j}^{(1)}\right)}\left(\left\|\mathbf{v}-\mathbf{v}_{h}\right\|_{L^{2}\left(\Omega_{j}^{(2)}\right)}+\left|q-q_{h}\right|_{c, \Omega_{j}^{(2)}}\right) \\
& \quad \leq C h d_{j}^{N / 2}\left(\left\|\mathbf{v}-\mathbf{v}_{h}\right\|_{L^{2}\left(\Omega_{j}^{(2)}\right)}+\left|q-q_{h}\right|_{c, \Omega_{j}^{(2)}}\right) .
\end{aligned}
$$

In the last two steps in (3.34), we have used the $H^{2}$ a priori regularity $\|w\|_{H^{2}(\Omega)} \leq$ $C\|\varphi\|_{L^{2}\left(\Omega_{j}^{(1)}\right)}$ and the inequality

$$
\|\varphi\|_{L^{2}\left(\Omega_{j}^{(1)}\right)} \leq C d_{j}^{N / 2}\|\varphi\|_{L^{\infty}(\Omega)} \leq C d_{j}^{N / 2} .
$$

Using the formula (2.7) of the bilinear form $b$ and similar to (3.26), we have

$$
\begin{aligned}
b_{\Omega_{j}^{(2)}}\left(\boldsymbol{\Phi}-\Pi^{h} \boldsymbol{\Phi}, q-q_{h}\right) \\
=-\sum_{K \in \mathcal{J}_{h}} \int_{K \cap \Omega_{j}^{(2)}}\left(\boldsymbol{\Phi}-\Pi^{h} \boldsymbol{\Phi}\right) \nabla\left(q-Q^{h} q\right) d x \\
\quad+\sum_{e \in \Gamma_{h}} \int_{e \cap \Omega_{j}^{(2)}}\left\{\boldsymbol{\Phi}-\Pi^{h} \boldsymbol{\Phi}\right\}\left[q-q_{h}\right] d s \\
\quad-\sum_{e \in \Gamma_{h}^{0}} \int_{e \cap \Omega_{j}^{(2)}} \lambda_{2}\left[q-q_{h}\right]\left[\boldsymbol{\Phi}-\Pi_{h} \boldsymbol{\Phi}\right] d s .
\end{aligned}
$$

By the Cauchy-Schwarz inequality, the $H^{2}$ a priori regularity and the inequality (3.35), we have the following estimate for the first term in (3.36):

$$
\begin{aligned}
& \sum_{K \in \mathcal{J}_{h}} \int_{K \cap \Omega_{j}^{(2)}}\left(\boldsymbol{\Phi}-\Pi^{h} \mathbf{\Phi}\right) \nabla\left(q-Q^{h} q\right) d x \\
& \quad \leq C h^{1+r}\|w\|_{H^{2}\left(\Omega_{j}^{(3)}\right)}\|q\|_{H^{1+r}\left(\Omega_{j}^{(3)}\right)} \\
& \quad \leq C h^{1+r}\|\varphi\|_{L^{2}\left(\Omega_{j}^{(1)}\right)}\|q\|_{H^{1+r}\left(\Omega_{j}^{(3)}\right)} \leq C h^{1+r} d_{j}^{N / 2}\|q\|_{H^{1+r}\left(\Omega_{j}^{(3)}\right)}
\end{aligned}
$$

and, by (2.14),

$$
\begin{aligned}
\text { (3.38) } \sum_{e \in \Gamma_{h}} \int_{e \cap \Omega_{j}^{(2)}}\left\{\boldsymbol{\Phi}-\Pi^{h} \boldsymbol{\Phi}\right\}\left[q-q_{h}\right] d s & \leq C\left\|\boldsymbol{\Phi}-\Pi_{h} \boldsymbol{\Phi}\right\| \|_{L^{2}\left(\Omega_{j}^{(3)}\right)}\left|q-q_{h}\right|_{c, \Omega_{j}^{(2)}} \\
& \leq C h\|\varphi\|_{L^{2}\left(\Omega_{j}^{(1)}\right)}\left|q-q_{h}\right|_{c, \Omega_{j}^{(2)}} \\
& \leq C h d_{j}^{N / 2}\left|q-q_{h}\right|_{c, \Omega_{j}^{(2)}}
\end{aligned}
$$




$$
\sum_{e \in \Gamma_{h}^{0}} \int_{e \cap \Omega_{j}^{(2)}} \lambda_{2}\left[q-q_{h}\right]\left[\boldsymbol{\Phi}-\Pi_{h} \boldsymbol{\Phi}\right] d s \leq C h d_{j}^{N / 2}\left|q-q_{h}\right|_{c, \Omega_{j}^{(2)}} .
$$

Substituting (3.37), (3.38) and (3.39) in (3.36), we arrive at the following estimate for the second term of $I_{2}$ :

$$
b_{\Omega_{j}^{(2)}}\left(\mathbf{\Phi}-\Pi^{h} \mathbf{\Phi}, q-q_{h}\right) \leq C h^{1+r} d_{j}^{N / 2}\|q\|_{H^{1+r}\left(\Omega_{j}^{(3)}\right)}+C h d_{j}^{N / 2}\left|q-q_{h}\right|_{c, \Omega_{j}^{(2)}} .
$$

Likewise for the third term of $I_{2}$, we have

$$
b_{\Omega_{j}^{(2)}}\left(\mathbf{v}-\mathbf{v}_{h}, w-Q^{h} w\right) \leq C h^{1+r} d_{j}^{N / 2}\|\mathbf{v}\|_{H^{r}\left(\Omega_{j}^{(3)}\right)}+C h d_{j}^{N / 2}\left\|\mathbf{v}-\mathbf{v}_{h}\right\|_{a, \Omega_{j}^{(2)}} .
$$

On combining (3.34), (3.40) and (3.41), we have

$$
\begin{aligned}
I_{2} \leq & C h^{1+r} d_{j}^{N / 2}\left(\|q\|_{H^{1+r}\left(\Omega_{j}^{(3)}\right)}+\|\mathbf{v}\|_{H^{r}\left(\Omega_{j}^{(3)}\right)}\right) \\
& +C h d_{j}^{N / 2}\left(\left\|\mathbf{v}-\mathbf{v}_{h}\right\|_{a, \Omega_{j}^{(2)}}+\left|q-q_{h}\right|_{c, \Omega_{j}^{(2)}}\right) .
\end{aligned}
$$

Finally, the estimates (3.33) and (3.42) imply the desired result of the lemma. The proof is complete.

Lemma 3.4. Let $g_{z} \in H_{0}^{1}(\Omega)$ be the solution of (3.2), $\mathbf{G}_{z}=\nabla g_{z}$, and $\left(g_{z, h}, \mathbf{G}_{z, h}\right) \in$ $W^{h} \times \mathbf{V}^{h}$ satisfy (3.3). Then for $0 \leq s \leq r-1$, we have

$$
\left|g_{z}-g_{z, h}\right|_{c, 1, \Omega, z,-s}+\left\|\mathbf{G}_{z}-\mathbf{G}_{z, h}\right\|_{a, 1, \Omega, z,-s} \leq C h|\ln h|^{\bar{s}},
$$

where $\bar{s}=0$ if $0 \leq s<r-1$ and $\bar{s}=1$ if $s=r-1$.

Proof. Let $M>1$ be a real number to be determined later in this proof and let $J$ be an integer such that $M h \leq 2^{-J}$. Then $J \leq C \ln (1 / h)$. For notational convenience, set $E_{g}=g_{z}-g_{z, h}$ and $E_{\mathbf{G}}=\mathbf{G}_{z}-\mathbf{G}_{z, h}$. In view of $\Omega=B_{M h}(z) \cup\left(\bigcup_{j=0}^{J} \Omega_{j}\right)$ and the triangle inequality, we have

$$
\begin{aligned}
& \left|E_{g}\right|_{c, 1, \Omega, z,-s} \leq\left|E_{g}\right|_{c, 1, B_{M h}(z), z,-s}+\sum_{j=0}^{J}\left|E_{g}\right|_{c, 1, \Omega_{j}, z,-s}, \\
& \left\|E_{\mathbf{G}}\right\|_{a, 1, \Omega, z,-s} \leq\left\|E_{\mathbf{G}}\right\|_{a, 1, B_{M h}(z), z,-s}+\sum_{j=0}^{J}\left\|E_{\mathbf{G}}\right\|_{a, 1, \Omega_{j}, z,-s} .
\end{aligned}
$$

By the definitions of the norms $|\cdot|_{c, 1, \Omega_{j}, z,-s}$ and $|\cdot|_{a, 1, \Omega_{j}, z,-s}$ and the CauchySchwarz inequality, we have for $0 \leq j \leq J$

$$
\begin{aligned}
& \left|E_{g}\right|_{c, 1, \Omega_{j}, z,-s}+\left\|E_{\mathbf{G}}\right\|_{a, 1, \Omega_{j}, z,-s} \\
= & \sum_{e \in \Gamma_{h}} \int_{e \cap \Omega_{j}} \sigma_{z, h}^{-s}\left|\left[E_{g}\right]\right| d s+\sum_{e \in \Gamma_{h}^{0}} \int_{e \cap \Omega_{j}} h_{e} \sigma_{z, h}^{-s}\left|\left[E_{\mathbf{G}}\right]\right| d s+\int_{\Omega_{j}} \sigma_{z, h}^{-s}\left|E_{\mathbf{G}}\right| d x \\
\leq & C d_{j}^{N / 2+s} h^{-s}\left(\left|E_{g}\right|_{c, \Omega_{j}}+\left\|E_{\mathbf{G}}\right\|_{a, \Omega_{j}}\right)
\end{aligned}
$$

and

$$
\begin{aligned}
& \left|E_{g}\right|_{c, 1, B_{M h}(z), z,-s}+\left\|E_{\mathbf{G}}\right\|_{a, 1, B_{M h}(z), z,-s} \\
& \quad \leq C(M h)^{N / 2+s} h^{-s}\left(\left|E_{g}\right|_{c, B_{M h}(z)}+\left\|E_{\mathbf{G}}\right\|_{a, B_{M h}(z)}\right) \\
& \quad \leq C M^{N / 2+s} h^{N / 2+1}\left\|g_{z}\right\|_{H^{2}(\Omega)} \leq C M^{N / 2+s} h .
\end{aligned}
$$


In (3.47), we have used the result in Lemma 2.2 and the a priori regularity $\left\|g_{z}\right\|_{H^{2}(\Omega)}$ $\leq C\left\|\delta_{z}\right\|_{L^{2}\left(K_{z}\right)} \leq C h^{-N / 2}$. Next, applying the local error estimates in Lemma 2.3 for the two norms $\left|E_{g}\right|_{c, \Omega_{j}}$ and $\left\|E_{\mathbf{G}}\right\|_{a, \Omega_{j}}$ on the right-hand side of (3.46) and then using Lemma 3.2, we get

$$
\begin{aligned}
\left|E_{g}\right|_{c, 1, \Omega_{j}, z,-s}+\left\|E_{\mathbf{G}}\right\|_{a, 1, \Omega_{j}, z,-s} & \\
\leq & C d_{j}^{N / 2+s} h^{r-s}\left\|g_{z}\right\|_{H^{1+r}\left(\Omega_{j}^{(1)}\right)} \\
& +C d_{j}^{s-1+\varepsilon} h^{-s-\varepsilon}\left(\left|E_{g}\right|_{L^{1}\left(\Omega_{j}\right)}+\left\|E_{\mathbf{G}}\right\|_{W^{-1,1}\left(\Omega_{j}\right)}\right) \\
\leq & C d_{j}^{1-r+s} h^{r-s}+C d_{j}^{s-1+\varepsilon} h^{-s-\varepsilon}\left(\left|E_{g}\right|_{L^{1}\left(\Omega_{j}\right)}+\left\|E_{\mathbf{G}}\right\|_{W^{-1,1}\left(\Omega_{j}\right)}\right) .
\end{aligned}
$$

From (3.44), 3.45), 3.47) and (3.48), we deduce that

$$
\left|E_{g}\right|_{c, 1, \Omega, z,-s}+\left\|E_{\mathbf{G}}\right\|_{a, 1, \Omega, z,-s} \leq C M^{N / 2+s} h+C h \Theta(r-1-s)+L_{1}+L_{2},
$$

where $\Theta$ is defined in (3.21) and

$$
\begin{gathered}
L_{1}=C h^{-1}\left\|E_{g}\right\|_{L^{1}(\Omega), z, 1-s-\varepsilon}, \\
L_{2}=C \sum_{j=0}^{J} d_{j}^{s-1+\varepsilon} h^{-s-\varepsilon}\left\|E_{\mathbf{G}}\right\|_{W^{-1,1}\left(\Omega_{j}\right)} .
\end{gathered}
$$

By a similar procedure, it follows that

$$
\begin{aligned}
& \left\|E_{g}\right\|_{L^{1}(\Omega), z, 1-s-\varepsilon} \leq\left\|E_{g}\right\|_{L^{1}\left(B_{M h}(z)\right), z, 1-s-\varepsilon}+\sum_{j=0}^{J}\left\|E_{g}\right\|_{L^{1}\left(\Omega_{j}\right), z, 1-s-\varepsilon} \\
& \leq C M^{N / 2+s} h^{N / 2}\left\|E_{g}\right\|_{L^{2}(\Omega)}+C \sum_{j=0}^{J} d_{j}^{s-1+\varepsilon} h^{1-s-\varepsilon}\left\|E_{g}\right\|_{L^{1}\left(\Omega_{j}\right)} \\
& \leq C h^{2} M^{N / 2+s}+C \sum_{j=0}^{J} d_{j}^{s-1+\varepsilon} h^{1-s-\varepsilon}\left\|E_{g}\right\|_{L^{1}\left(\Omega_{j}\right)},
\end{aligned}
$$

which implies

$$
L_{1}+L_{2} \leq C M^{N / 2+s} h+C \sum_{j=0}^{J} d_{j}^{s-1+\varepsilon} h^{-s-\varepsilon}\left(\left\|E_{g}\right\|_{L^{1}\left(\Omega_{j}\right)}+\left\|E_{\mathbf{G}}\right\|_{W^{-1,1}\left(\Omega_{j}\right)}\right)
$$

We are now in a position to estimate $\left\|E_{g}\right\|_{L^{1}\left(\Omega_{j}^{(1)}\right)}$ and $\left\|E_{\mathbf{G}}\right\|_{W^{-1,1}\left(\Omega_{j}^{(1)}\right)}$. Recall the following formulas:

$$
\begin{gathered}
\left\|E_{g}\right\|_{L^{1}\left(\Omega_{j}^{(1)}\right)}=\sup _{\substack{\varphi \in C_{0}^{\infty}\left(\Omega_{j}^{(1)}\right) \\
\|\varphi\|_{L^{\infty}\left(\Omega_{j}^{(1)}\right)}=1}}\left(E_{g}, \varphi\right), \\
\left\|E_{\mathbf{G}}\right\|_{W^{-1,1}\left(\Omega_{j}^{(1)}\right)}=\sup _{\substack{\psi \in C_{0}^{\infty}\left(\Omega_{j}^{(1)}\right)^{N} \\
\|\psi\|_{W^{1, \infty}\left(\Omega_{j}^{(1)}\right)}=1}}\left(E_{\mathbf{G}}, \psi\right) .
\end{gathered}
$$

For any $\varphi \in C_{0}^{\infty}\left(\Omega_{j}^{(1)}\right)$ satisfying $\|\varphi\|_{L^{\infty}\left(\Omega_{j}^{(1)}\right)}=1$, let $w \in H_{0}^{1}(\Omega) \cap H^{2}(\Omega)$ be the solution of

$$
-\Delta w=\varphi \quad \text { in } \Omega .
$$


Then letting $\mathbf{\Phi}=\nabla w$, we have for any $q \in W^{h}, \mathbf{v} \in \mathbf{V}^{h}$

$$
\begin{aligned}
a(\boldsymbol{\Phi}, \mathbf{v})+b(\mathbf{v}, w) & =0, \\
-b(\boldsymbol{\Phi}, q)+c(w, q) & =(\varphi, q) .
\end{aligned}
$$

By a straightforward manipulation, we obtain

$$
\begin{aligned}
\left(E_{g}, \varphi\right)= & -b\left(\boldsymbol{\Phi}-\Pi_{h} \mathbf{\Phi}, E_{g}\right)+c\left(w-Q^{h} w, E_{g}\right) \\
& +a\left(E_{\mathbf{G}}, \Pi_{h} \mathbf{\Phi}-\mathbf{\Phi}\right)+b\left(E_{\mathbf{G}}, Q^{h} w-w\right) .
\end{aligned}
$$

By applying Lemma 3.3 and 3.2 it follows that

$$
\begin{aligned}
\left(E_{g}, \varphi\right) \leq & C h^{1+r}|\ln h|^{\bar{r}} d_{j}^{1-r}+C h^{r} d_{j}^{1-r}\left(\left|E_{g}\right|_{c, 1, \Omega}+\left\|E_{\mathbf{G}}\right\|_{a, 1, \Omega}\right) \\
& +C h d_{j}^{N / 2}\left(\left\|E_{\mathbf{G}}\right\|_{a, \Omega_{j}^{(2)}}+\left|E_{g}\right|_{c, \Omega_{j}^{(2)}}\right)
\end{aligned}
$$

where $\bar{r}=0$ if $r>1$ and $\bar{r}=1$ if $r=1$. On the other hand, for any $\psi \in C_{0}^{\infty}\left(\Omega_{j}^{(1)}\right)^{N}$ satisfying $\|\psi\|_{W^{1, \infty}\left(\Omega_{j}^{(1)}\right)}=1$, if $w \in H_{0}^{1}(\Omega) \cap H^{2}(\Omega)$ is the solution of

$$
-\Delta w=\nabla \cdot \psi \quad \text { in } \Omega,
$$

then letting $\mathbf{\Phi}=\nabla w+\psi$, similar to the derivation of (2.8) we have

$$
\begin{aligned}
& a(\Phi, \mathbf{v})+b(\mathbf{v}, w)=(\psi, \mathbf{v}), \quad \forall \mathbf{v} \in H_{h}^{2}(\Omega)^{N}, \\
& -b(\Phi, q)+c(w, q)=0, \quad \forall q \in H_{h}^{2}(\Omega) .
\end{aligned}
$$

With a straightforward manipulation, we obtain

$$
\begin{aligned}
\left(E_{\mathbf{G}}, \psi\right)= & b\left(\boldsymbol{\Phi}-\Pi_{h} \mathbf{\Phi}, E_{g}\right)-c\left(w-Q^{h} w, E_{g}\right) \\
& -a\left(E_{\mathbf{G}}, \Pi_{h} \mathbf{\Phi}-\mathbf{\Phi}\right)-b\left(E_{\mathbf{G}}, Q^{h} w-w\right) .
\end{aligned}
$$

Using Lemma 3.3 and 3.2 again, we get

$$
\begin{aligned}
\left(E_{\mathbf{G}}, \psi\right) \leq & C h^{1+r}|\ln h|^{\bar{r}} d_{j}^{1-r}+C h^{r} d_{j}^{1-r}\left(\left|E_{g}\right|_{c, 1, \Omega}+\left\|E_{\mathbf{G}}\right\|_{a, 1, \Omega}\right) \\
& +C h d_{j}^{N / 2}\left(\left\|E_{\mathbf{G}}\right\|_{a, \Omega_{j}^{(2)}}+\left|E_{g}\right|_{c, \Omega_{j}^{(2)}}\right) .
\end{aligned}
$$

Inserting the two estimates (3.55) and 3.57 in 3.51) and (3.52), respectively, yields

$$
\begin{aligned}
\left\|E_{g}\right\|_{L^{1}\left(\Omega_{j}\right)}+\left\|E_{\mathbf{G}}\right\|_{W^{-1,1}\left(\Omega_{j}\right)} \\
\leq \quad C h^{1+r}|\ln h|^{r} d_{j}^{1-r}+C h^{r} d_{j}^{1-r}\left(\left|E_{g}\right|_{c, 1, \Omega}+\left\|E_{\mathbf{G}}\right\|_{a, 1, \Omega}\right) \\
\quad+C h d_{j}^{N / 2}\left(\left\|E_{\mathbf{G}}\right\|_{a, \Omega_{j}^{(2)}}+\left|E_{g}\right|_{c, \Omega_{j}^{(2)}}\right),
\end{aligned}
$$

which, when the local error estimate in Lemma 2.3 is applied to the last term

$$
\begin{aligned}
& \left\|E_{\mathbf{G}}\right\|_{a, \Omega_{j}^{(2)}}+\left|E_{g}\right|_{c, \Omega_{j}^{(2)}} \leq C h^{r} d_{j}^{1-r-N / 2} \\
& \quad+C d_{j}^{-N / 2-1}\left(\frac{h}{d_{j}}\right)^{-\varepsilon}\left(\left\|E_{g}\right\|_{L^{1}\left(\Omega_{j}^{(3)}\right)}+d_{j}\left\|E_{\mathbf{G}}\right\|_{L^{1}\left(\Omega_{j}^{(3)}\right)}\right),
\end{aligned}
$$

results in

$$
\begin{aligned}
& \left\|E_{g}\right\|_{L^{1}\left(\Omega_{j}\right)}+\left\|E_{\mathbf{G}}\right\|_{W^{-1,1}\left(\Omega_{j}\right)} \\
& \leq \quad C h^{1+r}|\ln h|^{\bar{r}} d_{j}^{1-r}+C h^{r} d_{j}^{1-r}\left(\left|E_{g}\right|_{c, 1, \Omega}+\left\|E_{\mathbf{G}}\right\|_{a, 1, \Omega}\right) \\
& \quad+\left(\frac{h}{d_{j}}\right)^{1-\varepsilon}\left(\left\|E_{g}\right\|_{L^{1}\left(\Omega_{j}^{(3)}\right)}+d_{j}\left\|E_{\mathbf{G}}\right\|_{L^{1}\left(\Omega_{j}^{(3)}\right)}\right) .
\end{aligned}
$$


We are now about to insert (3.60) into (3.50). Before we write the result of this insertion, we note that the contribution of the last term of (3.60) to (3.50) is

$$
\begin{aligned}
& \sum_{j=0}^{J} d_{j}^{s-1+\varepsilon} h^{-s-\varepsilon}\left(\frac{h}{d_{j}}\right)^{1-\varepsilon}\left(\left\|E_{g}\right\|_{L^{1}\left(\Omega_{j}^{(3)}\right)}+d_{j}\left\|E_{\mathbf{G}}\right\|_{L^{1}\left(\Omega_{j}^{(3)}\right)}\right) \\
& \leq C h^{-1} \sum_{j=0}^{J}\left(\frac{h}{d_{j}}\right)^{1-\varepsilon}\left\|E_{g}\right\|_{L^{1}\left(\Omega_{j}^{(3)}\right), z, 1-s-\varepsilon} \\
& \quad+C \sum_{j=0}^{J}\left(\frac{h}{d_{j}}\right)^{1-2 \varepsilon}\left\|E_{\mathbf{G}}\right\|_{L^{1}\left(\Omega_{j}^{(3)}\right), z,-s} \\
& \leq C h^{-1} \Theta(1-\varepsilon)\left\|E_{g}\right\|_{L^{1}(\Omega), z, 1-s-\varepsilon}+C \Theta(1-2 \varepsilon)\left\|E_{\mathbf{G}}\right\|_{a, 1, \Omega, z,-s} .
\end{aligned}
$$

Hence, inserting (3.60) into (3.50) and using (3.61), we obtain

$$
\begin{aligned}
L_{1}+L_{2} \leq & C M^{N / 2+s} h+\Theta(r-s-\varepsilon) h|\ln h|^{\bar{r}} \\
& +\Theta(r-s-\varepsilon)\left(\left|E_{g}\right|_{c, 1, \Omega}+\left\|E_{\mathbf{G}}\right\|_{a, 1, \Omega}\right) \\
& +C \Theta(1-\varepsilon) L_{1}+C \Theta(1-2 \varepsilon)\left\|E_{\mathbf{G}}\right\|_{a, 1, \Omega, z,-s}
\end{aligned}
$$

Since $1-\varepsilon>0$, using (3.21), we can choose $M$ sufficiently large so that $\Theta(1-\varepsilon)$ is small enough for the term $C \Theta(1-\varepsilon) L_{1}$ on the right-hand side of (3.62) to be absorbed into the left-hand side. Then we insert (3.62) into (3.49) to get

$$
\begin{aligned}
&\left\|E_{g}\right\|_{c, 1, \Omega, z,-s}+\left|E_{\mathbf{G}}\right|_{a, 1, \Omega, z,-s} \\
& \leq \quad C M^{N / 2+s} h+C h \Theta(r-1-s)+\Theta(r-s-\varepsilon) h|\ln h|^{\bar{r}} \\
&+\Theta(r-s-\varepsilon)\left(\left|E_{g}\right|_{c, 1, \Omega}+\left\|E_{\mathbf{G}}\right\|_{a, 1, \Omega}\right) \\
&+C \Theta(1-2 \varepsilon)\left\|E_{\mathbf{G}}\right\|_{a, 1, \Omega, z,-s},
\end{aligned}
$$

which, when the last term is eliminated by means of taking $M$ sufficiently large, leads to the following estimate:

$$
\begin{aligned}
\left\|E_{g}\right\|_{c, 1, \Omega, z,-s}+\left|E_{\mathbf{G}}\right|_{a, 1, \Omega, z,-s} \\
\leq \quad C M^{N / 2+s} h+C h \Theta(r-1-s)+\Theta(r-s-\varepsilon) h|\ln h|^{\bar{r}} \\
\quad+C \Theta(r-s-\varepsilon)\left(\left|E_{g}\right|_{c, 1, \Omega}+\left\|E_{\mathbf{G}}\right\|_{a, 1, \Omega}\right) .
\end{aligned}
$$

In particular, (3.63) holds true for $s=0$, which gives us

$$
\begin{aligned}
\left\|E_{g}\right\|_{c, 1, \Omega}+\left|E_{\mathbf{G}}\right|_{a, 1, \Omega} \\
\leq \quad C M^{N / 2} h+C h \Theta(r-1)+\Theta(r-\varepsilon) h|\ln h|^{\bar{r}} \\
\quad+\Theta(r-\varepsilon)\left(\left|E_{g}\right|_{c, 1, \Omega}+\left\|E_{\mathbf{G}}\right\|_{a, 1, \Omega}\right) .
\end{aligned}
$$

Eliminating the last term in (3.64) with a sufficiently large $M$, one can see that

$$
\begin{aligned}
\left\|E_{g}\right\|_{c, 1, \Omega}+\left|E_{\mathbf{G}}\right|_{a, 1, \Omega} & \leq C M^{N / 2} h+C h \Theta(r-1)+\Theta(r-\varepsilon) h|\ln h|^{\bar{r}} \\
& \leq C h|\ln h|^{\bar{r}} .
\end{aligned}
$$

On substituting (3.65) into (3.63), we have

$$
\begin{aligned}
&\left\|E_{g}\right\|_{c, 1, \Omega, z,-s}+\left|E_{\mathbf{G}}\right|_{a, 1, \Omega, z,-s} \\
& \leq \quad C M^{N / 2+s} h+C h \Theta(r-1-s)+C \Theta(r-s-\varepsilon) h|\ln h|^{\bar{r}} \\
& \quad+C h \Theta(r-s-\varepsilon)|\ln h|^{\bar{r}} .
\end{aligned}
$$


If $0 \leq s<r-1$, then $\bar{r}=0$ and $r-s-\varepsilon>0$. Thus, from (3.66) we conclude the desired (3.43). The proof is complete.

\section{Pointwise ERror estimate for the vector approximation}

The main result of this section is the pointwise error estimate for the vector approximation in Theorem 4.1

Theorem 4.1. Let $(p, \mathbf{u})$ and $\left(p_{h}, \mathbf{u}_{h}\right)$ satisfy (2.10) and $0 \leq s \leq r-1$. Then there is a constant $C>0$ such that for any $z \in \bar{\Omega}$, we have

$$
\left|\left(\mathbf{u}-\mathbf{u}_{h}\right)(z)\right| \leq C|\ln h|^{\bar{s}}\left(h^{-1}\left\|p-Q^{h} p\right\|_{L^{\infty}(\Omega), z, s}+\left\|\mathbf{u}-\Pi^{h} \mathbf{u}\right\|_{L^{\infty}(\Omega), z, s}\right),
$$

where $\bar{s}=0$ if $0 \leq s<r-1$ and $\bar{s}=1$ if $s=r-1$.

Proof. Let $K_{z} \in \mathcal{J}_{h}$ be such that $z \in \bar{K}_{z}$. Let $\boldsymbol{\delta}_{z} \in C_{0}^{1}\left(K_{z}\right)^{N}$ be the vector function whose components are $\delta_{z}$ defined in the proof of Theorem 3.1. Then it follows that

$$
\left\|\nabla \cdot\left(\boldsymbol{\delta}_{z}\right)\right\|_{L^{t}(\Omega)} \leq C h^{-1-N / t^{\prime}}, \quad 1 \leq t \leq \infty, \frac{1}{t}+\frac{1}{t^{\prime}}=1 .
$$

Similar to (3.1), we have

$$
\left|\left(\mathbf{u}-\mathbf{u}_{h}\right)(z)\right| \leq C\left\|\mathbf{u}-\Pi^{h} \mathbf{u}\right\|_{L^{\infty}(\Omega), z, s}+\left|\left(\boldsymbol{\delta}_{z}, \mathbf{u}-\mathbf{u}_{h}\right)\right| .
$$

Let $\tilde{g}_{z} \in H_{0}^{1}(\Omega)$ be the solution of

$$
-\Delta \tilde{g}_{z}=\nabla \cdot\left(\boldsymbol{\delta}_{z}\right) .
$$

Further let $\tilde{\mathbf{G}}_{z}=\nabla \tilde{g}_{z}+\boldsymbol{\delta}_{z}$. Then $\nabla \cdot \tilde{G}_{z}=0$. Also let $\left(\tilde{g}_{z, h}, \tilde{\mathbf{G}}_{z, h}\right) \in W^{h} \times \mathbf{V}^{h}$ be the finite element approximation of $\left(\tilde{g}_{z}, \tilde{\mathbf{G}}_{z}\right)$ satisfying

$$
\begin{aligned}
a\left(\tilde{\mathbf{G}}_{z}-\tilde{\mathbf{G}}_{z, h}, \mathbf{v}\right) & +b\left(\mathbf{v}, \tilde{g}_{z}-\tilde{g}_{z, h}\right)=0 \\
-b\left(\tilde{\mathbf{G}}_{z}-\tilde{\mathbf{G}}_{z, h}, q\right) & +c\left(\tilde{g}_{z}-\tilde{g}_{z, h}, q\right)=0
\end{aligned}
$$

for any $(q, \mathbf{v}) \in W^{h} \times \mathbf{V}^{h}$. Then

$$
\begin{aligned}
\left(\boldsymbol{\delta}_{z}, \mathbf{u}-\mathbf{u}_{h}\right)= & a\left(\tilde{\mathbf{G}}_{z}, \mathbf{u}-\mathbf{u}_{h}\right)+b\left(\mathbf{u}-\mathbf{u}_{h}, \tilde{g}_{z}\right) \\
= & a\left(\tilde{\mathbf{G}}_{z}-\tilde{\mathbf{G}}_{z, h}, \mathbf{u}-\Pi^{h} \mathbf{u}\right)+b\left(\mathbf{u}-\Pi^{h} \mathbf{u}, \tilde{g}_{z}-\tilde{g}_{z, h}\right) \\
& +b\left(\tilde{\mathbf{G}}_{z}-\tilde{\mathbf{G}}_{z, h}, Q^{h} p-p\right)+c\left(\tilde{g}_{z},-\tilde{g}_{z, h}, p-Q^{h} p\right) .
\end{aligned}
$$

By the same arguments as those used in the proof of Theorem 3.1, we have

$$
\begin{aligned}
\left(\boldsymbol{\delta}_{z}, \mathbf{u}-\right. & \left.\mathbf{u}_{h}\right) \\
\leq & \left(h^{-1}\left\|p-Q^{h} p\right\|_{L^{\infty}(\Omega), z, s}+\left\|\mathbf{u}-\Pi^{h} \mathbf{u}\right\|_{L^{\infty}(\Omega), z, s}\right) \\
& \cdot\left(\left\|\nabla\left(\tilde{g}_{z}-Q^{h} \tilde{g}_{z}\right)\right\|_{L^{1}(\Omega), z,-s}+\left\|\nabla \cdot\left(\tilde{\mathbf{G}}_{z}-\Pi^{h} \tilde{\mathbf{G}}_{z}\right)\right\|_{L^{1}(\Omega), z,-s}\right. \\
& \left.+\left|\tilde{g}_{z}-\tilde{g}_{z, h}\right|_{c, 1, \Omega, z,-s}+\left\|\tilde{\mathbf{G}}_{z}-\tilde{\mathbf{G}}_{z, h}\right\|_{a, 1, \Omega, z,-s}\right) .
\end{aligned}
$$

Using the results in Lemma 4.1 and 4.2 , we obtain the desired estimate of the theorem.

Lemma 4.1. Let $\tilde{g}_{z} \in H_{0}^{1}(\Omega)$ be the solution of (4.2). Then we have

$$
\left\|\tilde{g}_{z}\right\|_{H^{1+r}\left(\Omega_{j}^{(3)}\right)} \leq C h^{-1} d_{j}^{1-r-N / 2}
$$

and

$$
\left\|\nabla\left(\tilde{g}_{z}-Q^{h} \tilde{g}_{z}\right)\right\|_{L^{1}(\Omega), z,-s}+h\left\|\nabla \cdot\left(\tilde{\mathbf{G}}_{z}-\Pi^{h} \tilde{\mathbf{G}}_{z}\right)\right\|_{L^{1}(\Omega), z,-s} \leq C|\ln h|^{\bar{s}},
$$


where $\tilde{\mathbf{G}}_{z}=\nabla \tilde{g}_{z}, \bar{s}=1$ if $0 \leq s<r-1$ and $\bar{s}=1$ if $s=r-1$.

Lemma 4.2. Let $\tilde{g}_{z} \in H_{0}^{1}(\Omega)$ be the solution of (4.2), $\tilde{\mathbf{G}}_{z}=\nabla \tilde{g}_{z}$, and $\left(\tilde{g}_{z, h}, \tilde{\mathbf{G}}_{z, h}\right) \in$ $W^{h} \times \mathbf{V}^{h}$ satisfy (4.3). Then, for $0 \leq s \leq r-1$, we have

$$
\left|\tilde{g}_{z}-\tilde{g}_{z, h}\right|_{c, 1, \Omega, z,-s}+\left\|\tilde{\mathbf{G}}_{z}-\tilde{\mathbf{G}}_{z, h}\right\|_{a, 1, \Omega, z,-s} \leq C|\ln h|^{\bar{s}},
$$

where $\bar{s}=0$ if $0 \leq s<r-1$ and $\bar{s}=1$ if $s=r-1$.

The proofs of these two lemmas are almost the same as those of Lemmas 3.2 and 3.4. The only difference is that the right-hand side function for $\tilde{g}_{z}$ is $\nabla \cdot\left(\boldsymbol{\delta}_{z}\right)$ which gives an extra factor $h^{-1}$ for all bounds associated with $\tilde{g}_{z}$ and $\tilde{\mathbf{G}}_{z}$. In the proof of Lemma 4.2, we use the results of Lemma 4.1 So the corresponding terms on the right-hand sides of the inequalities derived in the proof of Lemma 3.2 are multiplied by the factor $h^{-1}$. We omit the details.

\section{REFERENCES}

[1] S. Agmon, A. Douglis and L. Nirenberg, Estimates near the boundary for solutions of elliptic partial differential equations satisfying general boundary conditions, I, Comm. Pure. Appl. Math., 12 (1959), 623-722. MR 23:A2610

[2] D. N. Arnold, An interior penalty finite element method with discontinuous elements, SIAM J. Numer. Anal., 19 (1982), 742-760. MR 83f:65173

[3] D. N. Arnold, F. Brezzi, B. Cockburn and L. D. Martin, Unified analysis of discontinuous Galerkin methods for elliptic problems, SIAM J. Numer. Anal., 39 (2002), 1749-1779. MR 2002k:65183

[4] I. Babuška, The finite element method with penalty, Math. Comp., 27 (1973), 221-228. MR 50:3607

[5] G. A. Baker, Finite element methods for elliptic equations using nonconforming elements, Math. Comp., 31 (1977), 45-59. MR 55:4737

[6] F. Bassi, S. ReBay, G. Mariotti, S. Pedinotti and M. Savini, A high-order accurate discontinuous finite element method for inviscid and viscous turbomachinery flows, Second European Conference on Turbomachinery fluid dynamics and thermodynamics (Antwerpen, Belgium) (R. Decuypere and G. Dibelius, eds.), Technologisch Institut, March 1997, 99-108.

[7] C. E. Baumann and J. T. Oden, A discontinuous hp finite element method for convectiondiffusion problems, Comput. Meth. Appl. Mech. Engrg., 175 (1999), 311-341. MR 2000d:65171

[8] F. Brezzi, G. Manzini, D. Marini, P. Pietra and A. Russo, Discontinuous Galerkin approximations for elliptic problems, Numer. Methods for Partial Differential Equations, 16 (2000), 365-378. MR 2001e:65178

[9] P. Castillo, B. Cockburn, I. Perugia and D. Schötzau, An a priori error analysis of the local discontinuous Galerkin method for elliptic problems, SIAM J. Numer. Anal. 38 (2000), 1676-1706. MR 2002k:65175

[10] H. Chen, Local error estimates of mixed discontinuous Galerkin methods for elliptic problems, J. Numer. Math., Vol. 12 (2004), 1-22.

[11] H. Chen, Z. Chen, Pointwise Error Estimates of Discontinuous Galerkin Methods with Penalty for Second-Order Elliptic Problems, SIAM J. Numer. Anal., 2004, to appear.

[12] Z. Chen, On the relationship of various discontinuous finite element methods for second order elliptic equations, East-West Numer. Math., 9 (2001), 99-122.

[13] B. Cockburn, S. Hou and C. W. Shu, TVB Runge-Kutta local projection discontinuous Galerkin finite element method for conservation laws IV: The multidimensional case, Math. Comp., 54 (1990), 545-581. MR 90k:65162

[14] B. Cockburn, G. E. Karniadakis and C. W. Shu, Discontinuous Galerkin Methods, Theory, Computation and Applications, Lecture Notes in Computational Science and Engineering, Vol. 11, Springer-Verlag, Berlin, 2000. MR 2002b:65004

[15] B. Cockburn and C. W. Shu, The local discontinuous finite element method for convection diffusion systems, SIAM J. Numer. Anal., 35 (1998), 2440-2463. MR 99j:65163 
[16] A. Demlow, Localized pointwise error estimates for mixed finite element methods, Math. Comp., 73 (2004) 1623-1653.

[17] J. Douglas and T. Dupont, Interior penalty procedures for elliptic and parabolic Galerkin methods, Lecture Notes in Physics, Vol. 58, Springer-Verlag, Berlin, 1976, 207-216. MR 55:13823

[18] J. T. Freund, The discontinuous Galerkin method for a scalar elliptic equation, in Computational mechanics: New trends and applications, S. Idehlson, E. Onate and E. Dvorkin (eds), CIMNE, 1998. MR 2002b:00032

[19] J. A. Nitsche, Über ein variationsprinzip zur lösung Dirichlet-problemen bei verwendung von teilräumen, die keinen randbedingungen unteworfen sind, abh. Math. Sem. Univ. Hamburg, 36 (1971), 9-15. MR 49:6649

[20] J. A. Nitche and A. H. Schatz, Interior estimates for Ritz-Galerkin methods, Math. Comp., 28 (1974), 937-958. MR 51:9525

[21] B. Riviére, M. F. Wheeler, and V. Girault, Improved energy estimates for interior penalty, constrained and discontinuous Galerkin methods for elliptic problems, I, Comput. Geosci., 3 (1999), 337-360. MR 2001d:65145

[22] A. H. Schatz, Pointwise error estimates and asymptotic error expansion inequalities for the finite element method on irregular grids: Part I. Global estimates, Math. Comp., 67 (1998), 877-899. MR 98j:65082

[23] A. H. Schatz and L. B. Wahlbin, Interior maximum norm estimates for finite element methods, Math. Comp., 31 (1977), 414-442. MR 55:4748

[24] A. H. Schatz and L. B. Wahlbin, Maximum norm estimates in the finite element method on plane polygonal domains. Part 1, Math. Comp., 32 (1978), 73-109. MR 58:19233a

[25] M. F. Wheeler, An elliptic collocation-finite element method with interior penalties, SIAM J. Numer. Anal., 15 (1978), 152-161. MR 57:11117

Department of Mathematics, University of Wyoming, Laramie, Wyoming 82070

E-mail address: hchen@uwyo.edu 